\title{
THE EQUIVARIANT HOMOTOPY TYPE OF $G$-ANR'S FOR PROPER ACTIONS OF LOCALLY COMPACT GROUPS
}

\author{
SERGEY A. ANTONYAN \\ Departamento de Matemáticas, Facultad de Ciencias \\ Universidad Nacional Autónoma de México \\ 04510 México Distrito Federal, México \\ E-mail: antonyan@unam.mx \\ ERIK ELFVING \\ Department of Mathematics and Statistics, P.O. Box 68 (Gustaf Hällströmin katu 2b) \\ FI-00014 University of Helsinki, Finland \\ E-mail: Erik.Elfving@helsinki.fi
}

\begin{abstract}
We prove that if $G$ is a locally compact Hausdorff group then every proper $G$-ANR space has the $G$-homotopy type of a $G$-CW complex. This is applied to extend the JamesSegal $G$-homotopy equivalence theorem to the case of arbitrary locally compact proper group actions.
\end{abstract}

1. Introduction. In this paper we study equivariant absolute neighborhood retracts or $G$-ANR's from the homotopical point of view, where the acting group $G$ is an arbitrary locally compact Hausdorff group and the actions are proper in the sense of R. Palais [32]. We prove the following:

MAIn TheOREM 1.1. Let $G$ be a locally compact group and $X$ a $G$-ANR. Then $X$ has the G-homotopy type of a $G$-CW complex.

For $G$ a compact group the theorem is proved in [9]. The reader can find in [9] also some additional relevant information concerning the earlier particular cases of this result.

Our proof of Main Theorem relies on the so called Approximate Slice Theorem established in [6], which enables us to generalize our proof in [9] to proper actions of arbitrary locally compact groups. On this base we first prove the key Lemma 5.1 which asserts that every $G$-ANR has arbitrarily fine $G$-normal covers.

2000 Mathematics Subject Classification: Primary 55P91; Secondary 54C55.

Key words and phrases: proper action, $G$-ANR, $G$-CW complex, $G$-nerve, $G$-homotopy type. The paper is in final form and no version of it will be published elsewhere. 
The $G$-nerve of such a cover is just the analogue of Waner's $G$-simplicial space (see [35]) associated with a tube-segmental $G$-cover. However, in our case the acting group $G$ is arbitrary locally compact (not necessarily Lie) and its quotients $G / H$, which are the " $G$-vertices" of a $G$-nerve, may have bad local structure in general; this leads in turn to a bad local structure of the proper $G$-nerve. The Approximate Slice Theorem just enables us to always work with only those " $G$-vertices" $G / H$ which are smooth proper $G$-manifolds (the corresponding compact subgroup $H \subset G$ is called large in the text), and this fact finally guarantees that the relevant $G$-nerves have the $G$-homotopy type of a $G$-CW complex. More precisely, we prove in Proposition 5.2 that the $G$-nerves of $G$-normal covers dominate arbitrary closely the given $G$-ANR space, and then using the $G$-manifold structure of the " $G$-vertices", we prove in Theorem 5.3 that these $G$ nerves have the $G$-homotopy type of a $G$-CW complex. The final step in our argument is Theorem 5.5 which claims that a $G$-space, which is $G$-dominated by a $G$-CW complex, has the $G$-homotopy type of a $G$-CW complex.

In Proposition 4.2 we show that the topology of the $G$-nerve behaves nicely with respect to the adjunction space construction. This enables one to represent the $G$-nerve as a limit of a filtration in which each of the spaces is obtained from the previous one by the adjunction space construction. This is one of the key points in the proof of the above mentioned Theorem 5.3.

As a corollary of our Main Theorem and Equivariant Whitehead Theorem (see Theorem 5.4 below) we obtain the following extension of the James-Segal Theorem [24], [25] to proper actions of arbitrary locally compact groups:

Corollary 1.2. Let $G$ be a locally compact group and $f: X \rightarrow Y$ a $G$-map between two $G$-ANR spaces. Then $f$ is a G-homotopy equivalence if and only if for every closed subgroup $H \subset G$ the restriction $f^{H}: X^{H} \rightarrow Y^{H}$ to the $H$-fixed point sets is an ordinary homotopy equivalence.

Corollary 1.3. Let $G$ be a locally compact group and $X$ a $G$-ANR space such that for every compact subgroup $H \subset G$, the $H$-fixed point set $X^{H}$ is contractible. Then $X$ is a $G$-AR.

2. Preliminaries. In this paper the letter $G$ will denote a locally compact Hausdorff topological group, unless stated otherwise. By $e$ we denote the unit element of $G$.

By a $G$-space we mean a completely regular Hausdorff topological space $X$ together with a fixed continuous action $G \times X \rightarrow X$ of the topological group $G$ on $X$. We shall denote by $g x$ the image of the pair $(g, x) \in G \times X$ under the action.

If $X$ is a $G$-space, then for a subset $S \subset X$ and a subgroup $H \subset G$, the $H$-hull (or $H$-saturation) of $S$ is defined by $H(S)=\{h s \mid h \in H, s \in S\}$. If $S$ is the one point set $\{x\}$, then the $H$-hull $H(S)$ is denoted by $H(x)$ and called the $H$-orbit of $x$. The $H$-orbit space is denoted by $X / H$.

A subset $S \subset X$ is called $H$-invariant if it coincides with its $H$-hull, i.e., $S=H(S)$. For any $x \in X$, the subgroup $G_{x}=\{g \in G \mid g x=x\}$ is called the stabilizer (or stationary subgroup) at $x$. If $G_{x}=G$ then $x$ is called a $G$-fixed point. Since we assume that $G$-spaces are Hausdorff spaces ( $T_{1}$ would suffice here), the isotropy subgroups are closed subsets 
of $G$. For a subgroup $H \subset G$, the set $X^{H}=\left\{x \in X \mid H \subset G_{x}\right\}$ is called the $H$-fixed point set of $X$.

If $X$ and $Y$ are two $G$-spaces, then a continuous map $f: X \rightarrow Y$ is called a $G$-map or an equivariant map if $f(g x)=g f(x)$ for every $x \in X$ and $g \in G$. If $G$ acts trivially on $Y$ then we use the term "invariant map" instead of "equivariant map". Any $G$-map $f: X \rightarrow Y$ sends the $H$-fixed point set $X^{H}$ into the $H$-fixed point set $Y^{H}$. We shall denote by $f^{H}$ the restriction $\left.f\right|_{X^{H}}: X^{H} \rightarrow Y^{H}$.

A homotopy $F: X \times I \rightarrow Y$ is called a $G$-homotopy if $F(g x, t)=g F(x, t)$ for every $x \in X, g \in G$ and $t \in I$. Two $G$-maps $f_{0}, f_{1}: X \rightarrow Y$ are $G$-homotopic if there exists a $G$-homotopy $F: X \times I \rightarrow Y$ such that $F(x, 0)=f_{0}(x)$ and $F(x, 1)=f_{1}(x)$ for all $x \in X$. We write $f_{0} \simeq_{G} f_{1}$ if $f_{0}$ and $f_{1}$ are $G$-homotopic. Two $G$-spaces $X$ and $Y$ are $G$-homotopy equivalent if there exist $G$-maps $f: X \rightarrow Y$ and $g: Y \rightarrow X$ such that $g \circ f \simeq_{G} \operatorname{id}_{X}$ and $f \circ g \simeq_{G} \operatorname{id}_{Y}$.

Let $\gamma$ be an open cover of $Y$. Then a $G$-homotopy $F: X \times I \rightarrow Y$ is said to be limited by $\gamma$, or simply, a $\gamma$-G-homotopy provided for any $x \in X$, there exists $\Gamma \in \gamma$ such that $F(x, t) \in \Gamma$ for all $t \in I$. In such a case $F(\cdot, 0)$ and $F(\cdot, 1)$ are called $\gamma$-G-homotopic G-maps.

We shall say that a $G$-space $X$ is $\gamma$ - $G$-dominated by a $G$-space $Y$ if there exist $G$-maps $f: X \rightarrow Y$ and $\varphi: Y \rightarrow X$ such that $\varphi f$ is $\gamma$-G-homotopic to the identity map id id $_{X}$ of.

If $X$ is a $G$-space and $A$ a closed $G$-invariant subset of $X$, the inclusion $i: A \rightarrow X$ is called a $G$-cofibration if it has the $G$-homotopy extension property for all $G$-maps $f: X \rightarrow Y$ and $G$-homotopies $\varphi: A \times I \rightarrow Y$ with $\varphi(a, 0)=f(a)$ for $a \in A$. More precisely, given $f$ and $\varphi$, there exists a $G$-homotopy $\phi: X \times I \rightarrow Y$ such that $\left.\phi\right|_{A \times I}=\varphi$ and $\phi(x, 0)=f(x)$. For basic properties of $G$-cofibrations see, for example, [13] and [16].

For a closed subgroup $H \subset G$, by $G / H$ we will denote the $G$-space of cosets $\{g H \mid$ $g \in G\}$ under the action induced by left translations.

A compatible metric $\rho$ on a $G$-space $X$ is called invariant or $G$-invariant if $\rho(g x, g y)=$ $\rho(x, y)$ for all $g \in G$ and $x, y \in X$.

In 1961 Palais [32] introduced the very important concept of a proper action of an arbitrary locally compact group $G$ and proved that for such actions slices still exist at each point, whenever $G$ is a Lie group. This makes it possible to extend a substantial part of the theory of compact Lie transformation groups to locally compact ones.

Let $X$ be a $G$-space. Two subsets $U$ and $V$ in $X$ are called thin relative to each other [32, Definition 1.1.1] if the set

$$
\langle U, V\rangle=\{g \in G \mid g U \cap V \neq \emptyset\}
$$

has compact closure in $G$. A subset $U$ of a $G$-space $X$ is called small if every point in $X$ has a neighborhood thin relative to $U$. A $G$-space $X$ is called proper (in the sense of Palais) if every point in $X$ has a small neighborhood. Each orbit in a proper $G$-space is closed, and each stabilizer is compact [32, Proposition 1.1.4]. Furthermore, if $X$ is a compact proper $G$-space, then $G$ has to be compact as well.

Clearly, if $G$ is compact, then every $G$-space is proper. In the case when $G$ is discrete and $X$ is locally compact, the notion of a proper action is the same as the classical notion of a properly discontinuous action. When $G=\mathbb{R}$, the additive group of the reals, proper 
$G$-spaces are precisely the dispersive dynamical systems with regular orbit space (see [10, Ch. IV]).

Important examples of proper $G$-spaces are the coset spaces $G / H$ with $H$ a compact subgroup of a locally compact group $G$. Other interesting examples can be found in [1], [6] and [26]. The reader is referred to [8] for a discussion of the relationship between Palais proper $G$-spaces and Bourbaki proper $G$-spaces.

In the present paper we are especially interested in the class $G-\mathcal{M}$ of all metrizable proper $G$-spaces $X$ that admit a $G$-invariant metric. Observe that in this case the orbit space $X / G$ is metrizable. Indeed, if $\rho$ is a $G$-invariant metric on a proper $G$-space $X$, then the formula

$$
\widetilde{\rho}(G(x), G(y))=\inf \left\{\rho\left(x^{\prime}, y^{\prime}\right) \mid x^{\prime} \in G(x), y^{\prime} \in G(y)\right\}
$$

defines a metric $\widetilde{\rho}$, compatible with the quotient topology of $X / G$ [32, Theorem 4.3.4].

It is well-known that for $G$ a compact group, the class $G$ - $\mathcal{M}$ coincides with the class of all metrizable $G$-spaces (see e.g., [3, Proposition 5]). A fundamental result of R. Palais [32, Theorem 4.3.4], states that if $G$ is any Lie group, then $G$ - $\mathcal{M}$ includes all separable metrizable proper $G$-spaces. The question of whether the separability can be omitted in this Palais' result, still remains open (even for $G=\mathbb{R}$ and $G=\mathbb{Z}$ ). We refer to [8] for a further discussion of this problem.

A $G$-space $Y$ is called a $G$-equivariant absolute neighborhood extensor (for the class $G$-M) (notation: $Y \in G$-ANE) if for any closed invariant subset $A \subset X$ of a $G$-space $X \in G$-M and any $G$-map $f: A \rightarrow Y$, there exist an invariant neighborhood $U$ of $A$ in $X$ and a $G$-map $\psi: U \rightarrow Y$ such that $\left.\psi\right|_{A}=f$. If, in addition, one can always take $U=X$, then we say that $Y$ is an $G$-equivariant absolute extensor (notation: $Y \in G$-AE). The map $\psi$ is called a $G$-extension of $f$.

A $G$-space $Y \in G$ - $\mathcal{M}$ is called a $G$-equivariant absolute neighborhood retract (for the class $G$-M) (notation: $Y \in G$-ANR) provided that for any closed $G$-embedding $Y \hookrightarrow X$ in a $G$-space $X \in G$-M , there exists a $G$-retraction $r: U \rightarrow Y$, where $U$ is an invariant neighborhood of $Y$ in $X$. If, in addition, one can always take $U=X$, then we say that $Y$ is a $G$-equivariant absolute retract (notation: $Y \in G$-AR).

We note that, in general, a metrizable $G$-ANE space $Y$ need not be a $G$-ANR, because it may not belong to the class $G$ - $\mathcal{M}$. But if $Y \in G$-M and $Y \in G$-ANE, then clearly $Y \in G$-ANR. The converse is also true: for $G$ an almost connected group it was proved in [5, Remark 5], and the general case is treated in [2].

Let us recall the well known definition of a slice [32, p. 305]:

Definition 2.1. Let $G$ be a group, $H$ a closed subgroup of $G$, and $X$ a $G$-space. An $H$-invariant subset $S \subset X$ is called an $H$-slice in $X$ if $G(S)$ is open in $X$ and there is a $G$-equivariant map $f: G(S) \rightarrow G / H$, called the slicing map, such that $S=f^{-1}(e H)$. The saturation $G(S)$ will be said to be a tubular set and the subgroup $H$ will be referred to as the slicing subgroup.

If $G(S)=X$ then $S$ is called a global $H$-slice of $X$.

One of the most powerful results in the theory of topological transformation groups states (see [32, Proposition 2.3.1]) that, if $X$ is a proper $G$-space with $G$ a Lie group, 
then for any point $x \in X$, there exists a $G_{x}$-slice $S$ in $X$ with $x \in S$. In general, when $G$ is not a Lie group, it is no longer true that a slice exists at each point of $X$ (see [4]). However an approximate version of the slice theorem is true which is based on the notion of a large subgroup of $G$ first introduced in [4] and [6].

A closed subgroup $H \subset G$ is called a large subgroup (see [6]) if there exists a closed normal subgroup $N \subset G$ such that $N \subset H$ and $G / N$ is a Lie group. A $G$-space $X$ is called a rich $G$-space [6] if for any point $x \in X$ and any neighborhood $U$ of $x$, there exists a point $y \in U$ such that the stabilizer $G_{y}$ is a large subgroup of $G$, and $G_{x} \subset G_{y}$.

It was established in [6] that for a closed subgroup $H \subset G$ the following properties are equivalent: (1) $H$ is a large subgroup, (2) the quotient $G / H$ is locally contractible, (3) $G / H$ is a $G$-ANR space, (4) $G / H$ is $G$-homeomorphic to a smooth $G$-manifold on which $G$ acts by diffeomorphisms.

Theorem 2.6 below contains more precise information concerning the last property (4).

The following result can be regarded as an approximate version of the well known Slice Theorem of R. Palais [32] for non-Lie group actions and is a crucial tool in our argument:

Theorem 2.2 (Approximate Slice Theorem [6]). Let $X$ be a proper $G$-space and $x \in X$. Then for any neighborhood $O$ of $x$ in $X$ there exist a compact large subgroup $K$ of $G$ with $G_{x} \subset K$, and a $K$-slice $S$ such that $x \in S \subset O$. Moreover, if $X$ is a rich $G$-space, then the $K$-slice $S$ can be chosen in such a way that $G_{s}=K$ for a point $s \in S$.

The following lemma is a well known result in the Theory of Lie groups (see e.g., [19, Ch. II, Theorem 4.2]):

LEMma 2.3. Let $\Gamma$ be a Lie group and $\Delta$ a closed subgroup of $\Gamma$. Then the quotient space $\Gamma / \Delta$ has a unique smooth structure with the property that the natural $\Gamma$-action on $\Gamma / \Delta$ induced by left translations is smooth.

LEMMA 2.4. Let $\Gamma$ be a locally compact group, $\Lambda$ a closed normal subgroup of $\Gamma$, and $\Delta$ a closed subgroup of $\Gamma$ such that $\Lambda \subset \Delta$. Let $f: \Gamma / \Delta \rightarrow \frac{\Gamma / \Lambda}{\Delta / \Lambda}$ be the natural map defined by $f(\gamma \Delta)=[\gamma \Lambda]$, where $[\gamma \Lambda]$ is the left coset of the subgroup $\Delta / \Lambda$ in $\Gamma / \Lambda$ that contains the element $\gamma \Lambda \in \Gamma / \Lambda$. Then:

1. $f$ is a $\Gamma / \Lambda$-equivariant homeomorphism.

2. If $\Gamma$ is a Lie group, then $f$ is a $\Gamma / \Lambda$-equivariant diffeomorphism.

3. If $\Gamma$ is a Lie group and $\Delta$ a normal subgroup of $\Gamma$, then $f$ is an isomorphism of Lie groups.

Proof. (1) is evident.

(2) Since $\Gamma$ acts smoothly and $\Lambda$ acts trivially on $\Gamma / \Delta$, we infer that the Lie group $\Gamma / \Lambda$ acts smoothly on $\Gamma / \Delta$. Now the result follows from (1) and the uniqueness part of Lemma 2.3 .

(3) It is clear that $f$ is an isomorphism of topological groups. Since both $\Gamma / \Delta$ and $\frac{\Gamma / \Lambda}{\Delta / \Lambda}$ are Lie groups, $f$ should be a diffeomorphism (see, e.g., [19, Ch. II, Theorem 2.6]). 
LEMMA 2.5. Let $G$ be a locally compact group and $N_{0}, \ldots, N_{k}$ compact normal subgroups of $G$ such that each quotient $G / N_{i}$ is a Lie group. Let $N$ be the intersection $N_{0} \cap \cdots \cap N_{k}$. Then $G / N$ is a Lie group too.

Proof. Because of compactness of the subgroup $N_{i}$ the quotient homomorphism $\pi_{i}: G \rightarrow$ $G / N_{i}$ is a closed map for all $0 \leq i \leq k$. This yields that the diagonal homomorphism $\pi: G \rightarrow G / N_{0} \times \cdots \times G / N_{k}$ defined by $\pi(g)=\left(\pi_{0}(g), \ldots, \pi_{k}(g), g \in G\right.$, is also a closed map [18, Proposition 2.3.30]. Observe that the kernel of $\pi$ is precisely the intersection $N=N_{0} \cap \cdots \cap N_{k}$. This yields quickly that the continuous isomorphism $\tilde{\pi}: G / N \rightarrow \pi(G)$ induced by $\pi$ is a closed map, and hence, a homeomorphism. Thus the quotient group $G / N$ is topologically isomorphic to the image $\pi(G)$. On the other hand $\pi(G)$, being a closed subgroup of the Lie group $G / N_{0} \times \cdots \times G / N_{k}$, is itself a Lie group (see e.g., [19, Ch. II, Theorem 2.3]). Thus $G / N$ is a Lie group.

THEOREM 2.6. Let $G$ be a locally compact group and $H_{0}, \ldots, H_{n}$ large subgroups of $G$. Assume that $N_{0}, \ldots, N_{n}$ are compact normal subgroups of $G$ such that for every $i=$ $0, \ldots, n, N_{i} \subset H_{i}$ and $G / N_{i}$ is a Lie group. Then the product $G / H_{0} \times \cdots \times G / H_{n}$ is a smooth manifold. Furthermore, if we denote $N:=N_{0} \cap \cdots \cap N_{n}$ then $G / N$ is a Lie group, and the action of $G / N$ on $G / H_{0} \times \cdots \times G / H_{n}$ defined by the following rule is a smooth action:

$$
\bar{g} N\left(g_{0} H_{0}, \ldots, g_{n} H_{n}\right)=\left(\bar{g} g_{0} H_{0}, \ldots, \bar{g} g_{n} H_{n}\right),
$$

where $\bar{g} N \in G / N$ and $g_{i} H_{i} \in G / H_{i}, i=0, \ldots, n$.

Proof. Clearly $N$ is a compact normal subgroup of $G$. That the quotient group $G / N$ is a Lie group follows from Lemma 2.5.

According to Lemma 2.4(1),

$$
G / H_{i} \text { is } G / N_{i} \text {-homeomorphic to } \frac{G / N_{i}}{H_{i} / N_{i}} .
$$

We will consider just the smooth structure on $G / H_{i}$ induced by this homeomorphism. Hence, the topological product $G / H_{0} \times \cdots \times G / H_{n}$ is a smooth manifold.

In order to prove that the above defined action of $G / N$ on $G / H_{0} \times \cdots \times G / H_{n}$ is smooth, it suffices to show that the action of $G / N$ on each $G / H_{i}, i=1, \ldots, n$, defined by

$$
\bar{g} N\left(g_{i} H_{i}\right)=\bar{g} g_{i} H_{i}, \quad \bar{g} N \in G / N, \quad g_{i} H_{i} \in G / H_{i},
$$

is smooth.

Since $N_{i} / N$ is a closed subgroup of the Lie group $G / N$ the quotient group $\frac{H_{i} / N}{N_{i} / N}$ is a Lie group as well (see e.g., [19, Ch. II, Theorem 4.2]). On the other hand $G / N$ is also a Lie group, and by Lemma 2.4(1), the natural isomorphism $f: G / N_{i} \rightarrow \frac{G / N}{N_{i} / N}$ is a $G / N$-equivariant homeomorphism. Since the smooth structure on a Lie group is unique (see e.g., [19, Ch. II, Corollary 2.2]) we conclude that $f$ is, in fact a diffeomorphism. Thus $f: G / N_{i} \rightarrow \frac{G / N}{N_{i} / N}$ is a $G / N$-equivariant smooth isomorphism. Next, we observe that $f$ maps the closed subgroup $H_{i} / N_{i}$ onto $\frac{H_{i} / N}{N_{i} / N}$. In other words,

the pair $\left(G / N_{i}, H_{i} / N_{i}\right)$ is smoothly isomorphic to the pair $\left(\frac{G / N}{N_{i} / N}, \frac{H_{i} / N}{N_{i} / N}\right)$. 
This yields that the quotient

$$
G / H_{i}=\frac{G / N_{i}}{H_{i} / N_{i}} \quad \text { is } G / N_{i} \text {-diffeomorphic to } \frac{(G / N) /\left(N_{i} / N\right)}{\left(H_{i} / N\right) /\left(N_{i} / N\right)} .
$$

Now, by applying Lemma $2.4(2)$ to $\Gamma=G / N, \Lambda=N_{i} / N$ and $\Delta=H_{i} / N$, we get that

$$
\frac{(G / N) /\left(N_{i} / N\right)}{\left(H_{i} / N\right) /\left(N_{i} / N\right)} \quad \text { is } G / N \text {-diffeomorphic to } \frac{G / N}{H_{i} / N} \text {. }
$$

Thus,

$$
G / H_{i} \quad \text { is } G / N \text {-diffeomorphic to } \frac{G / N}{H_{i} / N} \text {. }
$$

Since by Lemma 2.3, the induced action of the Lie group $G / N$ on its quotient space $\frac{G / N}{H_{i} / N}$ is smooth, we infer that $G / H_{i}$ is a smooth $G / N$-space. This completes the proof.

3. G-CW complexes. In this chapter we present several results on $G$-CW complexes which are needed later. For the definition and basic properties of $G$-CW complexes we refer to [20], [21], [29], [27], [13] and [16]. We first recall some basic properties of adjunction spaces.

Let $X$ be a $G$-space, $A$ a closed invariant subset of $X$, and $f: A \rightarrow Y$ a $G$-map in a $G$-space $Y$. We denote the adjunction space by $X \cup_{f} Y$ and the natural projection by $p: X \sqcup Y \rightarrow X \cup_{f} Y$ (see [15, Section VI.6]). From the $G$-actions on $X$ and $Y$ the adjunction space inherits a natural $G$-action. Continuity of this action follows from the fact that in the commutative diagram

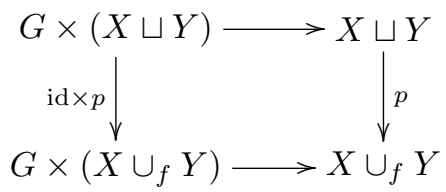

the map id $\times p$ is a quotient map, since $p$ is a quotient map and $G$ is locally compact (see [14, Lemma V.2.13], where a quotient map is called an identification map). Thus $X \cup_{f} Y$ is a $G$-space if it is a Hausdorff space. Note that the adjunction space of two Hausdorff spaces is not necessarily Hausdorff. We have, for example, the following results:

Proposition 3.1. If the spaces $X$ and $Y$ are normal topological spaces then the space $X \cup_{f} Y$ is a normal and, in particular, Hausdorff space.

Proof. See [15, VII.3.4].

Proposition 3.2. Let $X$ and $Y$ be Hausdorff $(G$-)spaces, $A$ a closed $(G$-)subspace of $X$ and $f: A \rightarrow Y$ a (G-)map. If the inclusion $A \subset X$ is a (G-)cofibration then the adjunction space $X \cup_{f} Y$ is a Hausdorff space.

Proof. See [16, Proposition 1.11].

If $X$ is a $G$-CW complex, the skeletons of $X$ are denoted by $X^{k}, k=0,1, \ldots$ A $G$-map $f: X \rightarrow Y$, where $X$ and $Y$ are $G$-CW complexes, is called skeletal if $f\left(X^{k}\right) \subset Y^{k}$ for 
all $k \geq 0$. Recall the following skeletal approximation theorem from [21, Theorem I.2.14, p. 31] (see also [28, Theorem 4.4] for the absolute case):

Theorem 3.3. Any $G$-map $f: X \rightarrow Y$ between $G$-CW complexes is $G$-homotopic to a skeletal $G$-map $\tilde{f}: X \rightarrow Y$. If $f$ is skeletal on a $G$-subcomplex $X_{0}$ of $X$ then $f$ is $G$-homotopic relative to $X_{0}$ to a skeletal $G$-map.

In the previous theorem the $G$-map $\tilde{f}$ is called a skeletal approximation or a cellular approximation to $f$.

Concerning properness of a $G$-CW complex we have the following result (see [27, p. 18]):

Proposition 3.4. A G-CW complex $X$ is a proper $G$-space if and only if $G_{x}$ is compact for each $x \in X$.

The following three propositions are not difficult to verify.

Proposition 3.5. Let $L$ and $A$ be proper $G$-CW complexes, $B \subset A$ a $G$-subcomplex and $\alpha: B \rightarrow L$ a cellular $G$-map. Denote $\alpha_{n}=\left.\alpha\right|_{B^{n}}: B^{n} \rightarrow L^{n}, n \geq 0$. Then $\bar{L}=A \cup_{\alpha} L$ is a proper $G$-CW complex with skeletons

$$
\bar{L}^{n}=A^{n} \cup_{\alpha_{n}} L^{n}
$$

and $L$ is a G-subcomplex of $\bar{L}$.

Proposition 3.6. Let $L_{0} \subset L_{1} \subset \cdots$ be a sequence of proper $G$-CW complexes such that $L_{n}$ is a $G$-subcomplex of $L_{n+1}$ for each $n \geq 0$. If we give the set $L=\bigcup_{n \geq 0} L_{n}$ the weak topology with respect to the family $\left\{L_{n}\right\}_{n \geq 0}$, then $L$ is a proper $G$-CW complex with skeletons

$$
L^{k}=\bigcup_{n \geq 0} L_{n}^{k}, \quad k=0,1, \ldots
$$

The product of two ordinary $\mathrm{CW}$ complexes need not be a $\mathrm{CW}$ complex in a natural way (see the footnote in [34, p. 401]). With $G$-CW complexes the situation is even more complicated [13, p. 103]. However, we have the following result (compare also [21, Proposition I.2.6, p. 23]):

Proposition 3.7. Let $X$ be a locally compact proper $G$-CW complex, $Y$ a locally compact $\mathrm{CW}$ complex endowed with the trivial G-action, and $B \subset Y$ a subcomplex. Then the product $X \times Y$ is a proper $G$-CW complex with skeletons

$$
(X \times Y)^{n}=\bigcup_{k+p=n} X^{k} \times Y^{p}
$$

and $X \times B$ is a $G$-subcomplex of $X \times Y$.

Next we consider the following commutative diagram of $G$-spaces and $G$-maps:

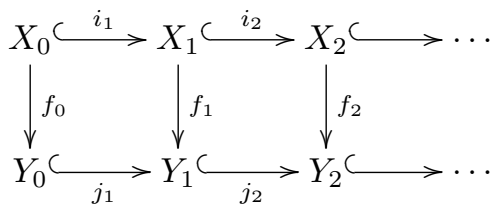


where the inclusions $i_{1}, i_{2}, \ldots$ and $j_{1}, j_{2}, \ldots$ are $G$-cofibrations and the maps $f_{0}, f_{1}, \ldots$ are $G$-homotopy equivalences. Let $X:=\bigcup X_{k}$ and $Y:=\bigcup Y_{k}$, endowed with the weak topologies with respect to the families $\left\{X_{k}\right\}_{k \geq 0}$ and $\left\{Y_{k}\right\}_{k \geq 0}$, respectively. The spaces $X$ and $Y$ are Hausdorff spaces by [16, Proposition 1.12]. The $G$-actions on all the spaces $X_{k}$ and $Y_{k}$ define $G$-actions on the spaces $X$ and $Y$. Continuity of these actions follows from the fact that the product $G \times X$ and $G \times Y$ have the weak topologies with respect to the families $\left\{G \times X_{k}\right\}_{k \geq 0}$ and $\left\{G \times Y_{k}\right\}_{k \geq 0}$, respectively, since $G$ is a locally compact Hausdorff space. The maps $f_{k}$ define a $G$-map $f: X \rightarrow Y$.

The proof of the following theorem is analogous to the proof of the non-equivariant case proved in [12].

TheOrem 3.8. The map $f: X \rightarrow Y$ is a G-homotopy equivalence.

THEOREM 3.9. Let $\left(A_{n}, B_{n}\right), n=1,2, \ldots$, be a sequence of proper $G$-CW pairs. Let $K_{0}$ be a proper $G$-CW complex and suppose that for all $n=1,2, \ldots$, the $G$-space $K_{n}$ is obtained from $K_{n-1}$ as an adjunction space

$$
K_{n}=A_{n} \cup_{\varphi_{n}} K_{n-1}
$$

where $\varphi_{n}: B_{n} \rightarrow K_{n-1}$ is a G-map. If we denote $K:=\bigcup_{n=0}^{\infty} K_{n}$ and give $K$ the weak topology with respect to the family $\left\{K_{n}\right\}_{n \geq 1}$, then the $G$-space $K$ has the $G$-homotopy type of a $G$-CW complex.

Proof. The actions $\Phi_{n}: G \times K_{n} \rightarrow K_{n}$ on the spaces $K_{n}$ together define an action $\Phi$ on the union $K=\bigcup_{n=0}^{\infty} K_{n}$. Since $K$ has the weak topology with respect to the family $\left\{K_{n}\right\}_{n \geq 0}$, the natural map $\iota: \bigsqcup_{n=0}^{\infty} K_{n} \rightarrow K$ is a quotient map. Thus, the map $\mathrm{id} \times \iota: G \times \bigsqcup_{n=0}^{\infty} K_{n} \rightarrow G \times K$ is a quotient map since $G$ is locally compact. Now continuity of $\Phi$ follows from the commutative diagram:

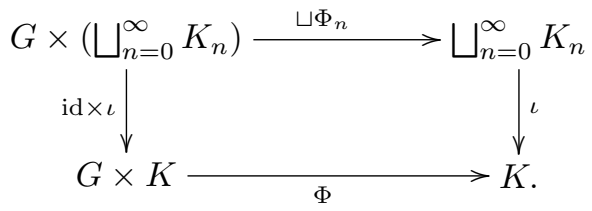

We shall construct a sequence $L_{0}, L_{1}, \ldots$ of proper $G$-CW complexes and $G$-homotopy equivalences $f_{n}: K_{n} \rightarrow L_{n}, n=0,1, \ldots$ First we define $L_{0}=K_{0}$ and $f_{0}=$ id. Suppose $n \geq 1$ and we have constructed $L_{0}, \ldots, L_{n-1}$ and $f_{0}, \ldots, f_{n-1}$. Let $\mu_{n}$ be a $G$-cellular approximation to the composite $G$-map $f_{n-1} \circ \varphi_{n}: B_{n} \rightarrow K_{n-1} \rightarrow L_{n-1}$, and define

$$
L_{n}:=A_{n} \cup_{\mu_{n}} L_{n-1} .
$$

By Proposition $3.5, L_{n}$ is a proper $G$-CW complex.

Let

$$
\alpha: A_{n} \cup_{\varphi_{n}} K_{n-1} \rightarrow A_{n} \cup_{f_{n-1} \varphi_{n}} L_{n-1}
$$

be the canonical extension of the $G$-map $f_{n-1}$, that is, the $G$-map induced by $f_{n-1}$ and the identity map on $A_{n}$. Since $f_{n-1}$ is a $G$-homotopy equivalence, also $\alpha$ is a $G$-homotopy equivalence by [22, Proposition 3.3]. 
Since $f_{n-1} \circ \varphi_{n} \simeq_{G} \mu_{n}$, by [22, Proposition 3.1] there is a $G$-homotopy equivalence

$$
\beta: A_{n} \cup_{f_{n-1} \varphi_{n}} L_{n-1} \rightarrow A_{n} \cup_{\mu_{n}} L_{n-1}
$$

with $\left.\beta\right|_{L_{n-1}}=\operatorname{id}_{L_{n-1}}$.

Then $f_{n}:=\beta \circ \alpha: K_{n} \rightarrow L_{n}$ is a $G$-homotopy equivalence extending $f_{n-1}$. Thus we obtain a commutative diagram

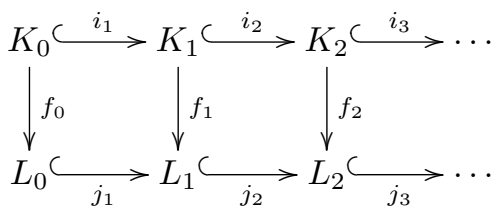

in which the inclusions are $G$-cofibrations and the maps $f_{0}, f_{1}, \ldots$ are $G$-homotopy equivalences.

Define $L:=\bigcup_{n>0} L_{n}$ and give $L$ the weak topology with respect to the family $\left\{L_{n}\right\}_{n \geq 0}$. By Proposition 3.6, $L$ is a proper G-CW complex. By Theorem 3.8, the map $f: K \rightarrow L$ induced by the maps $f_{n}$, is a $G$-homotopy equivalence.

The following lemma is easy to verify:

Lemma 3.10. Let $G$ be a locally compact group and $N$ a closed normal subgroup of $G$. Denote by $\pi: G \rightarrow G / N$ the natural homomorphism. Let $K$ be a closed subgroup (not necessarily normal) of $G / N$, and denote $H:=\pi^{-1}(K)$, which is a closed subgroup of $G$ containing $N$. Then the map

$$
\psi: G / H \rightarrow(G / N) / K ; \quad g H \mapsto \pi(g) K
$$

is a homeomorphism.

If we let $G$ act on the spaces $G / H$ and $(G / N) / K$ in the natural way, that is, the actions are induced by the left translations in $G$, then $\psi$ is also a $G$-map.

Proposition 3.11. Let $G$ be a locally compact group and $N$ a closed normal subgroup of $G$. Suppose $X$ is a $(G / N)$-CW complex with skeletons $\left\{X^{n}\right\}_{n \geq 0}$. Then $X$ is a $G$-CW complex with the same skeletons and equivariant cells.

Proof. The $G$-action on $X$ is given by

$$
G \times X \stackrel{\pi \times \mathrm{id}}{\longrightarrow}(G / N) \times X \rightarrow X .
$$

Let $\varphi: D^{n} \times(G / N) / K \rightarrow c$ be a characteristic $(G / N)$-map for a $(G / N)$-cell $c$. Define

$$
\bar{\varphi}=\varphi \circ(\mathrm{id} \times \psi): D^{n} \times G / H \rightarrow D^{n} \times(G / N) / K \rightarrow c,
$$

where the subgroup $H$ and the homeomorphism $\psi$ are as in the previous lemma. Then $\bar{\varphi}$ is a characteristic $G$-map for the $G$-cell $c$.

It is clear that the other conditions for $X$ being a $G$-CW complex are satisfied.

4. $G$-normal covers and $G$-nerves. In this chapter we introduce the concepts of a $G$-normal cover as in [6], and the $G$-nerve of a $G$-normal cover, following the ideas in [29] and $[35]$. 
General theory of simplicial sets can be found in [30]. Concrete examples can also be found in for example [33] and [35]. However, these constructions are not similar to ours, and compared with [30] we have no degeneracy operators. Hence we present the details of our construction in this chapter.

For any space $X$, the support of a map $f: X \rightarrow \mathbb{R}$ is the closed set

$$
\text { supp } f:=\overline{\{x \in X \mid f(x) \neq 0\}} \text {. }
$$

A family $\left\{\varphi_{\alpha} \mid \alpha \in \mathcal{A}\right\}$ of continuous maps $\varphi_{\alpha}: X \rightarrow I$ is called a partition of unity on $X$ if: (1) the supports of the $\varphi_{\alpha}$ form a locally finite closed cover of $X$ and, (2) $\sum_{\alpha} \varphi_{\alpha}(x)=1$ for each $x \in X$. If $\left\{U_{\beta} \mid \beta \in \mathcal{B}\right\}$ is a given open cover of $X$, we say that a partition $\left\{\varphi_{\beta} \mid \beta \in \mathcal{B}\right\}$ of unity is subordinated to $\left\{U_{\beta}\right\}$ if $\operatorname{supp} \varphi_{\beta} \subset U_{\beta}$ for all $\beta \in \mathcal{B}$.

If $\mathcal{U}$ is a cover of a space $X$ then for $A \subset X$ we denote

$$
\operatorname{St}(A, \mathcal{U})=\bigcup\{U \in \mathcal{U} \mid U \cap A \neq \emptyset\}
$$

the star of $A$ with respect to $\mathcal{U}$. A cover $\mathcal{U}$ of $X$ is a star-refinement of another cover $\mathcal{V}$ of $X$ if the cover $\{\operatorname{St}(U, \mathcal{U})\}_{U \in \mathcal{U}}$ is a refinement of $\mathcal{V}$.

A cover $\mathcal{U}$ of a $G$-space $X$ is $G$-invariant if each of the sets $U \in \mathcal{U}$ is $G$-invariant. A cover $\mathcal{U}$ of a $G$-space $X$ is a $G$-cover if $g U \in \mathcal{U}$ for every $U \in \mathcal{U}$ and $g \in G$. Note that the index set for a $G$-cover can be regarded as a $G$-set.

Let $\Lambda$ be a $G$-set and $\Lambda / G$ denote its orbit set. If $\mathcal{U}=\left\{U_{\lambda}\right\}_{\lambda \in \Lambda}$ is a $G$-cover of $X$ then for each $\mu \in \Lambda / G$, define

$$
\widetilde{U}_{\mu}:=\bigcup_{\lambda \in \mu} U_{\lambda} .
$$

In other words, if $\lambda \in \mu$ then we have $\widetilde{U}_{\mu}=G\left(U_{\lambda}\right)$. The $G$-invariant cover $\widetilde{\mathcal{U}}=\left\{\widetilde{U}_{\mu}\right\}_{\mu \in \Lambda / G}$ is called the saturation of $\mathcal{U}$.

Suppose $X \in G$-M. Let $H \subset G$ be a closed subgroup, $S$ an $H$-slice in $X$, and $O \subset G$ a neighborhood of the identity. The set $g O S$, where $g \in G$ and $g O S=\{g p s \mid p \in O, s \in S\}$, is called a tubular segment of type $H$, and the group $H$ is called its slicing group. The set $O S$ is called the initial tubular segment of length $O$ of the $H$-tube $G(S)$.

A family

$$
\mathcal{U}=\left\{g O_{\mu} S_{\mu} \mid g \in G, \mu \in \mathcal{M}\right\}
$$

consisting of tubular segments $g O_{\mu} S_{\mu}$ with compact large slicing subgroups $H_{\mu}$ is called a $G$-normal cover of $X$ if the family of open tubes $\widetilde{\mathcal{U}}=\left\{G\left(S_{\mu}\right) \mid \mu \in \mathcal{M}\right\}$ covers $X$ and there exists an invariant locally finite partition of unity $\left\{\varphi_{\mu}: X \rightarrow[0,1] \mid \mu \in \mathcal{M}\right\}$ subordinated to $\widetilde{\mathcal{U}}$.

A $G$-normal cover is called rich if each slicing subgroup $H_{\mu}$ is the stabilizer of a point $s_{\mu} \in S_{\mu}$. Note that the slices $S_{\mu}$ and the slicing subgroups $H_{\mu}$ form a part of the structure of a $G$-normal cover.

Next we construct the $G$-nerve of a $G$-normal cover.

Let $\mathcal{U}=\left\{g O_{\mu} S_{\mu} \mid g \in G, \mu \in \mathcal{M}\right\}$ be a $G$-normal cover of a space $X \in G$ - $\mathcal{M}$. Denote by $\Lambda=G \times \mathcal{M}$ the index set of the cover, and $\mathcal{U}=\left\{U_{\lambda}\right\}_{\lambda \in \Lambda}$, where $U_{\lambda}=g O_{\mu} S_{\mu}$ for $\lambda=(g, \mu)$. In what follows we shall identify $\mu \in \mathcal{M}$ with the orbit of $\lambda=(g, \mu)$ in $\Lambda$, 
and we will write $\lambda \in \mu$ whenever $\lambda=(g, \mu)$ for some $g \in G$. In particular, we have $\Lambda / G \approx \mathcal{M}$.

Denote $\widetilde{U}_{\mu}=G\left(S_{\mu}\right)$ and $\widetilde{\mathcal{U}}=\left\{\widetilde{U}_{\mu}\right\}_{\mu \in \mathcal{M}}$, the saturation of $\mathcal{U}$, and let $f_{\mu}: \widetilde{U}_{\mu} \rightarrow G / H_{\mu}$, $\mu \in \mathcal{M}$, be the slicing maps given by the definition of a slice.

Let $N=N(\widetilde{\mathcal{U}})$ denote the nerve of $\widetilde{\mathcal{U}}$, that is, a simplicial complex with vertices $\mu \in \mathcal{M}$ and simplexes defined by the following condition: $\left\{\mu_{0}, \ldots, \mu_{n}\right\}$ is a simplex of $N$ if and only if $\widetilde{U}_{\mu_{0}} \cap \cdots \cap \widetilde{U}_{\mu_{n}} \neq \emptyset$. Denote by $N_{n}=N_{n}(\widetilde{\mathcal{U}})$ the set of $n$-simplexes. We assume that $\mathcal{M}$ is well-ordered.

For each $n$-simplex $\sigma=\left\{\mu_{0}, \ldots, \mu_{n}\right\}, \mu_{0}<\cdots<\mu_{n}$, of $N$ we define an open subspace $K_{\sigma}$ of $G / H_{\mu_{0}} \times \cdots \times G / H_{\mu_{n}}$ as follows: If $\lambda_{0} \in \mu_{0}, \ldots, \lambda_{n} \in \mu_{n}$ are such that

$$
\bigcap_{i=0}^{n} U_{\lambda_{i}} \neq \emptyset
$$

then we consider the subset $f_{\mu_{0}}\left(U_{\lambda_{0}}\right) \times \cdots \times f_{\mu_{n}}\left(U_{\lambda_{n}}\right) \subset G / H_{\mu_{0}} \times \cdots \times G / H_{\mu_{n}}$. Then define $K_{\sigma}$ to be the union of such subsets, where the union is taken over those $n$-tuples of indices $\lambda_{0}, \ldots, \lambda_{n}$ that satisfy $(*)$ above. In other words,

$$
K_{\sigma}=\bigcup\left\{Q_{\lambda_{0}} \times \cdots \times Q_{\lambda_{n}} \mid \lambda_{i} \in \mu_{i}, i=0, \ldots, n, \bigcap_{i=0}^{n} U_{\lambda_{i}} \neq \emptyset\right\},
$$

where we denote $Q_{\lambda_{i}}:=f_{\mu_{i}}\left(U_{\lambda_{i}}\right) \subset G / H_{\mu_{i}}$. Observe that if $\lambda_{i}=\left(g, \mu_{i}\right)$ then $Q_{\lambda_{i}}=$ $g O_{\mu_{i}} H_{\mu_{i}}$. In particular, each set $Q_{\lambda_{i}}$ is open in $G / H_{\mu_{i}}$, and hence, $K_{\sigma}$ is an open $G$ invariant subset of the product $G / H_{\mu_{0}} \times \cdots \times G / H_{\mu_{n}}$.

Now, the $G$-action on $K_{\sigma}$ is defined by

$$
\left(g,\left(x_{0}, \ldots, x_{n}\right)\right) \mapsto\left(g x_{0}, \ldots, g x_{n}\right) .
$$

Next, we define a $G$-space $K_{*}(\mathcal{U})$, the simplicial $G$-nerve of $\mathcal{U}$ as follows.

The $n$-th space $K_{n}(\mathcal{U}), n \geq 0$, of $K_{*}(\mathcal{U})$ is

$$
K_{n}(\mathcal{U}):=\bigsqcup_{\sigma \in N_{n}} K_{\sigma}
$$

and the face operators $\partial_{i}: K_{n}(\mathcal{U}) \rightarrow K_{n-1}(\mathcal{U}), 0 \leq i \leq n$, are defined as follows.

Let $\sigma=\left\{\mu_{0}, \ldots, \mu_{n}\right\}, \mu_{0}<\cdots<\mu_{n}$, and $x=\left(x_{0}, \ldots, x_{n}\right) \in K_{\sigma} \subset K_{n}(\mathcal{U})$. Then $\tau_{i}=\left\{\mu_{0}, \ldots, \hat{\mu}_{i}, \ldots, \mu_{n}\right\}$, obtained from $\sigma$ by omitting $\mu_{i}$, is an $(n-1)$-simplex of $N$ and $\left(x_{0}, \ldots, \hat{x}_{i}, \ldots, x_{n}\right) \in K_{\tau_{i}}$. Now define

$$
\partial_{i}(x)=\left(x_{0}, \ldots, \hat{x}_{i}, \ldots, x_{n}\right) \in K_{\tau_{i}} .
$$

This gives us maps $\partial_{i}: K_{n}(\mathcal{U}) \rightarrow K_{n-1}(\mathcal{U}), 0 \leq i \leq n$.

The $G$-actions on the spaces $K_{\sigma}$ give us a $G$-action on every space $K_{n}(\mathcal{U}), n \geq 0$, and the face operators are equivariant maps.

Next we consider the discrete union

$$
\bigsqcup_{n \geq 0} K_{n}(\mathcal{U}) \times \Delta^{n}
$$

and the equivalence relation on it generated by

$$
\left(\partial_{i} x, t\right) \sim\left(x, \delta_{i} t\right)
$$


where $x \in K_{n}(\mathcal{U}), t \in \Delta^{n-1}, \delta_{i}\left(t_{0}, \ldots, t_{n-1}\right)=\left(t_{0}, \ldots, t_{i-1}, 0, t_{i}, \ldots, t_{n-1}\right) \in \Delta^{n}$ and $i \in\{0, \ldots, n\}, n \geq 1$.

We denote the quotient space by

$$
K(\mathcal{U})=\left|K_{*}(\mathcal{U})\right|=\left(\bigsqcup_{n \geq 0} K_{n}(\mathcal{U}) \times \Delta^{n}\right) / \sim .
$$

It is called the geometric $G$-nerve of $\mathcal{U}$.

Denote by $\pi: \bigsqcup_{n>0} K_{n}(\mathcal{U}) \times \Delta^{n} \rightarrow K(\mathcal{U})$ the projection.

The image of $\bigsqcup_{i=0}^{n} K_{i}(\mathcal{U}) \times \Delta^{i}$ under the map $\pi$ is called the n-skeleton of $K(\mathcal{U})$, and denoted by $K^{n}(\mathcal{U})$.

For $x=\left(x_{\mu_{0}}, \ldots, x_{\mu_{n}}\right) \in K_{\sigma} \subset G / H_{\mu_{0}} \times \cdots \times G / H_{\mu_{n}}$ and $t=\left(t_{0}, \ldots, t_{n}\right) \in \Delta^{n}$, denote $\pi(x, t):=\left|x_{\mu_{0}}, \ldots, x_{\mu_{n}} ; t_{0}, \ldots, t_{n}\right|=:|x ; t|$.

The $G$-actions on the spaces $K_{n}(\mathcal{U})$ fit together and give a $G$-action on $K(\mathcal{U})$ by $g\left|x_{\mu_{0}}, \ldots, x_{\mu_{n}} ; t_{0}, \ldots, t_{n}\right|=\left|g x_{\mu_{0}}, \ldots, g x_{\mu_{n}} ; t_{0}, \ldots, t_{n}\right|$. Since $G$ is locally compact the action on the quotient space is continuous.

Since there are no identifications between points of $K_{0}(\mathcal{U})$ we have that $K^{0}(\mathcal{U})=$ $K_{0}(\mathcal{U})=\bigsqcup_{\mu \in \Lambda / G} G / H_{\mu}$. Let $n \geq 1$. Each $\bar{t} \in \partial \Delta^{n}$ is of the form $\bar{t}=\delta_{i}(t)$ for some $t \in \Delta^{n-1}, i \in\{0, \ldots, n\}$. For each $n \geq 1$, we can define a continuous map $\alpha_{n}: K_{n}(\mathcal{U}) \times$ $\partial \Delta^{n} \rightarrow K^{n-1}(\mathcal{U})$ by

$$
\alpha_{n}\left(x, \delta_{i}(t)\right)=\left|\partial_{i} x ; t\right|
$$

In the commutative diagram

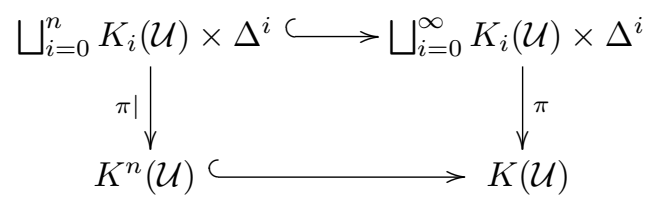

the map $\pi$ is a quotient map by definition.

The following proposition plays a key role in what follows:

Proposition 4.1. In the diagram (1) also the restriction map $\pi \mid$ is a quotient map.

Proof. Suppose $F \subset K^{n}(\mathcal{U})$ and $\pi^{-1}(F) \cap\left(K_{i}(\mathcal{U}) \times \Delta^{i}\right)$ is closed in $K_{i}(\mathcal{U}) \times \Delta^{i}$ for all $i=0, \ldots, n$, or equivalently,

$$
\pi^{-1}(F) \cap\left(K_{\sigma} \times \Delta^{\operatorname{dim}(\sigma)}\right) \text { is closed in } K_{\sigma} \times \Delta^{\operatorname{dim}(\sigma)}
$$

for all simplexes $\sigma$ of $N$ with $\operatorname{dim} \sigma \leq n$.

We prove that (2) holds for all simplexes $\sigma$ of $N$, from which it follows that $F$ is closed in $K(\mathcal{U})$ and thus in $K^{n}(\mathcal{U})$, which proves the proposition.

Let first $\sigma$ be an $(n+1)$-simplex of $N$ with the vertices $\mu_{0}, \ldots, \mu_{n+1}$. Denote $\sigma=$ $\left\{\mu_{0}, \ldots, \mu_{n+1}\right\}$. Now $F \subset K^{n}(\mathcal{U})$ and the identifications are such that each point of $K_{\sigma} \times \partial \Delta^{n+1}$ is identified with exactly one point of $K^{n}(\mathcal{U})$, and hence

$$
\pi^{-1}(F) \cap\left(K_{\sigma} \times \Delta^{n+1}\right) \subset K_{\sigma} \times \partial \Delta^{n+1} .
$$

Denote

$$
\Delta_{(i)}^{n}=\left\{\left(t_{0}, \ldots, t_{n+1}\right) \in \Delta^{n+1} \mid t_{i}=0\right\},
$$


the $i$-th face of $\Delta^{n+1}$, and $\tau_{i}=\left\{\mu_{0}, \ldots, \hat{\mu}_{i}, \ldots, \mu_{n+1}\right\}<\sigma$. The identifications on the $i$-th face of $K_{\sigma} \times \Delta^{n+1}$ can be given by the continuous map

$$
\begin{aligned}
\alpha_{i}^{\prime}: K_{\sigma} \times \Delta_{(i)}^{n} & \rightarrow K_{\tau_{i}} \times \Delta^{n} \\
\left(x_{0}, \ldots, x_{n+1} ; t_{0}, \ldots, t_{i-1}, 0, t_{i}, \ldots, t_{n}\right) & \mapsto\left(x_{0}, \ldots, \hat{x}_{i}, \ldots, x_{n+1} ; t_{0}, \ldots, t_{n}\right) .
\end{aligned}
$$

Now by the assumption (2) we have that

$$
A:=\pi^{-1}(F) \cap\left(K_{\tau_{i}} \times \Delta^{n}\right) \text { is closed in } K_{\tau_{i}} \times \Delta^{n}
$$

and the set

$$
B=\pi^{-1}(F) \cap\left(K_{\sigma} \times \Delta_{(i)}^{n}\right)
$$

is the inverse image of $A$ under the map $\alpha_{i}^{\prime}$, so it is closed in $K_{\sigma} \times \Delta_{(i)}^{n}$. Now $K_{\sigma} \times \Delta_{(i)}^{n}$ is closed in $K_{\sigma} \times \Delta^{n+1}$, and hence, $B$ is closed in $K_{\sigma} \times \Delta^{n+1}$. Since a simplex has only finitely many faces, the set $\pi^{-1}(F) \cap\left(K_{\sigma} \times \Delta^{n+1}\right)=\bigcup_{i=0}^{n+1} \pi^{-1}(F) \cap\left(K_{\sigma} \times \Delta_{(i)}^{n}\right)$ is closed in $K_{\sigma} \times \Delta^{n+1}$.

This proves the case $\operatorname{dim} \sigma=n+1$.

By induction we get (2) for all simplexes $\sigma$ of $N$. This completes the proof.

The previous proposition implies the following important

Proposition 4.2. The restriction

$$
\left(i_{n-1} \sqcup \pi\right) \mid: K^{n-1}(\mathcal{U}) \sqcup\left(K_{n}(\mathcal{U}) \times \Delta^{n}\right) \rightarrow K^{n}(\mathcal{U})
$$

is a quotient map, and thus, $K^{n}(\mathcal{U})$ is $G$-homeomorphic to the adjunction space

$$
\left(K_{n}(\mathcal{U}) \times \Delta^{n}\right) \cup_{\alpha_{n}} K^{n-1}(\mathcal{U}) .
$$

In the diagram

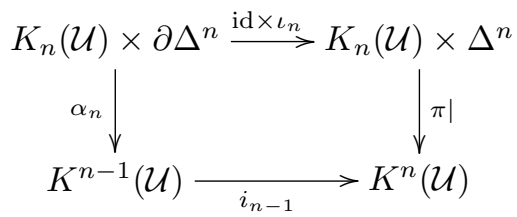

the map $\iota_{n}$ is a cofibration and thus id $\times \iota_{n}$ is a $G$-cofibration. On the other hand, $i_{n-1}: K^{n-1}(\mathcal{U}) \rightarrow K^{n}(\mathcal{U})$ is a $G$-cofibration by [13, Proposition II.1.2]. The spaces $K^{n}(\mathcal{U})$ are Hausdorff spaces by [16, Proposition 1.11].

The topology of $K(\mathcal{U})$ is the weak topology with respect to the filtration

$$
K^{0}(\mathcal{U}) \subset K^{1}(\mathcal{U}) \subset \cdots,
$$

and thus, $K(\mathcal{U})$ is a Hausdorff space (see [16, Proposition 1.12]).

Proposition 4.3. Let $\mathcal{U}=\left\{g O_{\mu} S_{\mu} \mid g \in G, \mu \in \mathcal{M}\right\}$ be a $G$-normal cover of a $G$-space $X$ with $f_{\mu}: \widetilde{U}_{\mu}=G\left(S_{\mu}\right) \rightarrow G / H_{\mu}, \mu \in \mathcal{M}$, the corresponding slicing maps. Choose a $G$-invariant locally finite partition of unity $\left\{\varphi_{\mu}\right\}_{\mu \in \mathcal{M}}$ subordinated to the open invariant cover $\widetilde{\mathcal{U}}=\left\{\widetilde{U}_{\mu}\right\}_{\mu \in \mathcal{M}}$. Define $P: X \rightarrow K(\mathcal{U})$ by

$$
P(x)=\left|f_{\mu_{0}}(x), \ldots, f_{\mu_{n}}(x) ; \varphi_{\mu_{0}}(x), \ldots, \varphi_{\mu_{n}}(x)\right|,
$$

where $x \in X$ and $\left\{\mu_{0}, \ldots, \mu_{n}\right\}=\left\{\mu \in \mathcal{M} \mid \varphi_{\mu}(x) \neq 0\right\}, \mu_{0}<\cdots<\mu_{n}$. Then $P$ is a continuous $G$-map. 
Proof. As above, we denote $\Lambda=G \times \mathcal{M}$ and identify the orbit set $\Lambda / G$ with $\mathcal{M}$. We shall write $\lambda \in \mu$ whenever there exists a $g \in G$ such that $\lambda=(g, \mu)$. Then $\mathcal{U}=\left\{U_{\lambda}\right\}_{\lambda \in \Lambda}$, where $U_{\lambda}=g O_{\mu} S_{\mu}$ for $\lambda=(g, \mu)$.

Denote $\sigma=\left\{\mu_{0}, \ldots, \mu_{n}\right\} \in N(\widetilde{\mathcal{U}})$. Since $\varphi_{\mu_{i}}(x)>0$ we see that $x \in \widetilde{U}_{\mu_{i}}$, and thus, there exists a $\lambda_{i} \in \mu_{i}$ for which $x \in U_{\lambda_{i}}, i=0, \ldots, n$. Consequently, $x \in \bigcap_{i=0}^{n} U_{\lambda_{i}}$, and hence, $\left(f_{\mu_{0}}(x), \ldots, f_{\mu_{n}}(x)\right) \in K_{\sigma}$ by the definition of $K_{\sigma}$. Thus $P(x) \in K(\mathcal{U})$.

To show the continuity of $P$ we first consider the following simplicial space. The $n$-th space $X_{\widetilde{\mathcal{U}}^{n}}$ is given by

$$
\bigsqcup_{\sigma \in N_{n}(\tilde{\mathcal{U}})} T_{\sigma}, \quad \text { where } T_{\sigma}=\bigcap_{\mu \in \sigma} \widetilde{U}_{\mu} \neq \emptyset,
$$

that is,

$$
X_{\widetilde{\mathcal{U}}^{n}}=\bigcup_{\sigma \in N_{n}(\widetilde{\mathcal{U}})}\left(T_{\sigma} \times\{\sigma\}\right) .
$$

The face maps are then defined as follows: if $n \geq 1,(x, \sigma) \in X_{\widetilde{\mathcal{U}}^{n}}$ and $i \in\{0, \ldots, n\}$, then $x \in T_{\sigma}$ and $T_{\sigma}$ is of the form $T_{\sigma}=\widetilde{U}_{\mu_{0}} \cap \cdots \cap \widetilde{U}_{\mu_{n}}$, where $\mu_{0}<\cdots<\mu_{n} \in \mathcal{M}$. Now define $\tau_{i}=\left\{\mu_{0}, \ldots, \hat{\mu}_{i}, \ldots, \mu_{n}\right\}$, that is, $\tau_{i}$ is obtained from $\sigma$ by omitting the $i$-th term, and by definition, $\partial_{i}(x, \sigma)=\left(x, \tau_{i}\right) \in X_{\widetilde{\mathcal{U}}^{n-1}}$.

As before, we define an equivalence relation on the space $\bigsqcup_{n \geq 0} X_{\widetilde{\mathcal{U}}^{n}} \times \Delta^{n}$ generated by the relations $\left(\partial_{i} x ; t\right) \sim\left(x ; \delta_{i} t\right)$, and consider the quotient space

$$
B X_{\widetilde{\mathcal{U}}}=\bigsqcup_{n \geq 0} X_{\widetilde{\mathcal{U}}^{n}} \times \Delta^{n} / \sim
$$

Denote by $\tilde{\pi}$ the projection $\bigsqcup_{n \geq 0} X_{\widetilde{\mathcal{U}}^{n}} \times \Delta^{n} \rightarrow B X_{\widetilde{\mathcal{U}}}$.

A $G$-action on $B X_{\widetilde{\mathcal{U}}}$ can be defined by

$$
g|(x, \sigma) ; t|=|(g x, \sigma) ; t| .
$$

Define a function $P^{\prime}: X \rightarrow B X_{\widetilde{\mathcal{U}}}$ as follows: If $x \in X$ and

$$
\left\{\mu_{0}, \ldots, \mu_{n}\right\}=\left\{\mu \in \Lambda / G \mid \varphi_{\mu}(x) \neq 0\right\}, \quad \mu_{0}<\cdots<\mu_{n},
$$

then denote $\sigma=\left\{\mu_{0}, \ldots, \mu_{n}\right\}$ and put

$$
P^{\prime}(x)=\left|(x, \sigma) ; \varphi_{\mu_{0}}(x), \ldots, \varphi_{\mu_{n}}(x)\right| .
$$

We now prove that $P^{\prime}$ is continuous. Let $V^{\prime}$ be a neighborhood of $x$ such that $\left.\varphi_{\mu}\right|_{V^{\prime}} \equiv 0$ for all but a finite number of $\mu$ 's. Let

$$
\left\{\beta_{0}, \ldots, \beta_{m}\right\}=\left\{\beta \in \mathcal{M} \mid \varphi_{\beta}(y) \neq 0 \text { for some } y \in V^{\prime}\right\} .
$$

If $x \notin \widetilde{U}_{\beta_{j}}$ for some $\beta_{j} \in\left\{\beta_{0}, \ldots, \beta_{m}\right\}$, we can find a neighborhood of $x$ in which $\varphi_{\beta_{j}} \equiv 0$; we exclude $\beta_{j}$ in this case. Thus we may assume that $x \in \widetilde{U}_{\beta_{j}}$ for all $j=0, \ldots, m$.

Assume $\beta_{0}<\cdots<\beta_{m}$. Now $V=\left(\bigcap_{j=0}^{m} \widetilde{U}_{\beta_{j}}\right) \cap V^{\prime}$ is a neighborhood of $x$. Let $\sigma^{\prime}=\left\{\beta_{0}, \ldots, \beta_{m}\right\}$. Then we can define

$$
\psi: V \rightarrow X_{\widetilde{\mathcal{U}}^{m}} \times \Delta^{m}
$$

by

$$
\psi(y)=\left(\left(y, \sigma^{\prime}\right), \varphi_{\beta_{0}}(y), \ldots, \varphi_{\beta_{m}}(y)\right), \quad y \in V
$$


which is clearly continuous. Now observe that

$$
\left.P^{\prime}\right|_{V}=\tilde{\pi} \circ \psi
$$

and hence, $P^{\prime}$ is continuous at $x$. That $P^{\prime}$ is a $G$-map follows from

$$
P^{\prime}(g x)=\left|(g x, \sigma) ; \varphi_{\mu_{0}}(g x), \ldots, \varphi_{\mu_{n}}(g x)\right|=\left|(g x, \sigma) ; \varphi_{\mu_{0}}(x), \ldots, \varphi_{\mu_{n}}(x)\right|=g P^{\prime}(x) .
$$

Next define a map

$$
\phi_{*}: X_{\widetilde{\mathcal{U}}^{*}}=\bigsqcup_{\substack{\sigma \in N_{n}(\widetilde{\mathcal{U}}) \\ n \geq 0}}\left(T_{\sigma} \times\{\sigma\}\right) \rightarrow K_{*}(\mathcal{U})
$$

as follows: if $(x, \sigma) \in X_{\widetilde{\mathcal{U}}^{n}}$ with $x \in T_{\sigma}=\widetilde{U}_{\mu_{0}} \cap \cdots \cap \widetilde{U}_{\mu_{n}}$ and $\mu_{0}<\cdots<\mu_{n} \in \mathcal{M}$, then we put

$$
\phi_{*}(x, \sigma)=\left(f_{\mu_{0}}(x), \ldots, f_{\mu_{n}}(x)\right) \in K_{\sigma} \subset K_{n}(\mathcal{U}) .
$$

Clearly $\phi_{*}$ is a continuous $G$-map.

Now $\phi_{*}$ satisfies

$$
\phi_{*}\left(\partial_{i}(x, \sigma)\right)=\partial_{i} \phi_{*}(x, \sigma),
$$

where $\partial_{i}$ on the left side denotes the face map in $X_{\widetilde{\mathcal{U}}^{*}}$, and on the right side the face map in $K_{*}(\mathcal{U})$. From this it follows that $\phi_{*}$ defines a continuous map

$$
\left|\phi_{*}\right|: B X_{\widetilde{\mathcal{U}}} \rightarrow K(\mathcal{U})
$$

such that the diagram

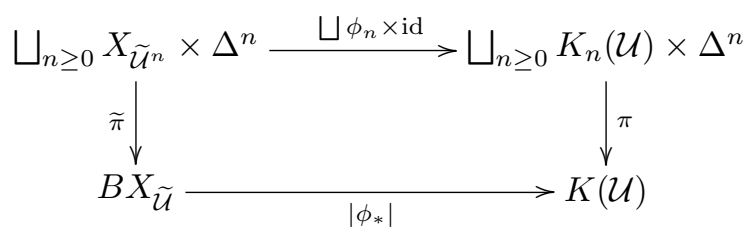

commutes.

Clearly $\left|\phi_{*}\right|$ is a $G$-map. Now $P=\left|\phi_{*}\right| \circ P^{\prime}: X \rightarrow B X_{\tilde{\mathcal{U}}} \rightarrow K(\mathcal{U})$, which proves that $P$ is a continuous $G$-map.

5. Proofs of the main theorem and corollaries. In this section we prove the main result formulated in Introduction. We start with the following key lemma:

Lemma 5.1. Let $G$ be a locally compact group and $X \in G$-M. Then for each open $G$-cover $\mathcal{V}$ of $X$, there exists a $G$-normal cover

$$
\mathcal{U}=\left\{g O_{t} R_{t} \mid g \in G, t \in T\right\}
$$

of $X$, accompanied with compact large slicing subgroups $H_{t}$, such that $\mathcal{U}$ is a starrefinement of $\mathcal{V}$. Furthermore, if $X$ is a rich $G$-space then $\mathcal{U}$ can be chosen to be rich, i.e., for each $t \in T$ there is a point $x_{t} \in R_{t}$ such that $G_{x_{t}}=H_{t}$.

Proof. Choose a $G$-invariant metric $d$ on $X$. We fix a point on each orbit $G(x) \subset X$, say $x \in G(x)$, and choose an element $V_{x} \in \mathcal{V}$ such that $x \in V_{x}$. Let $\varepsilon_{x}>0$ be such that $B\left(x, 5 \varepsilon_{x}\right) \subset V_{x}$, where $B(x, r)$ stands for the open ball of radius $r$ centered at $x$. 
By the continuity of the action of $G$ on $X$, there exist a neighborhood $Q_{x}$ of the unity in $G$ and a neighborhood $N_{x}$ of $x$ in $X$ such that $Q_{x} N_{x} \subset B\left(x, \varepsilon_{x}\right)$. By the Approximate Slice Theorem (see Theorem 2.2), there exist a compact large subgroup $H^{x} \subset G$ and an $H^{x}$-slice $S_{x}$ such that $x \in S_{x} \subset N_{x}$. Thus, we have $Q_{x} S_{x} \subset B\left(x, \varepsilon_{x}\right)$.

Let $\mathcal{W}$ be the totality of all these tubular segments $\left\{g Q_{x} S_{x} \mid g \in G\right\}$. Clearly, $\mathcal{W}$ is an open $G$-cover of $X$.

Let $p: X \rightarrow X / G$ be the orbit projection. Since $p(\mathcal{W})=\{p(W) \mid W \in \mathcal{W}\}$ is an open cover of the paracompact space $X / G$, there exists an open cover $\omega=\left\{\Omega_{t} \mid t \in T\right\}$ of $X / G$ which is a star-refinement of $p(\mathcal{W})$ (see [15, Ch. VIII, §3]).

We emphasize that all the covers we consider are essential, i.e., none of the elements of the cover is contained in the union of others.

It then follows that the invariant open cover $p^{-1}(\omega)=\left\{p^{-1}\left(\Omega_{t}\right) \mid t \in T\right\}$ of $X$ is a star-refinement of the invariant open cover $G(\mathcal{W})=\{G(W) \mid W \in \mathcal{W}\}$ of $X$. In particular, each element $p^{-1}\left(\Omega_{t}\right) \in p^{-1}(\omega)$ is a tubular set. Indeed, since $p^{-1}\left(\Omega_{t}\right)$ is a subset of a tubular set $G\left(S_{x}\right) \in G(\mathcal{W})$, the intersection $A=S_{x} \cap p^{-1}\left(\Omega_{t}\right)$ is a global $H^{x}$-slice of $p^{-1}\left(\Omega_{t}\right)$ and $Q_{x} A$ is a tubular segment of $p^{-1}\left(\Omega_{t}\right)$.

Next, for every index $t \in T$ we consider the set $\mathcal{L}_{t}$ of all tubular segments $O S=$ $\{g s \mid g \in O, s \in S\}$, where $O$ is a neighborhood of the unity of $G$ and $S$ is a global $H$ slice for $p^{-1}\left(\Omega_{t}\right)$ with $H$ a compact large subgroup of $G$. Observe that $\mathcal{L}_{t}$ is non-empty since $p^{-1}\left(\Omega_{t}\right)$ is a tubular set. Write

$$
\xi_{t}=\inf \left\{\operatorname{diam} O S \mid O S \in \mathcal{L}_{t}\right\} .
$$

We claim that $\xi_{t}=0$ iff $p^{-1}\left(\Omega_{t}\right)$ contains only one orbit. The "if" part is evident. Now assume that $\xi_{t}=0$. Then there are a point $a \in p^{-1}\left(\Omega_{t}\right)$ and a sequence of $H_{n}$-slices $S_{n}, n=1,2, \ldots$, containing the point $a$ such that $G\left(S_{n}\right)=p^{-1}\left(\Omega_{t}\right)$ and $\operatorname{diam} S_{n}<1 / n ; n=1,2, \ldots$ Pick a point $x \in p^{-1}\left(\Omega_{t}\right)$. As $p^{-1}\left(\Omega_{t}\right)=G\left(S_{n}\right)$ for every $n \geq 1$, there exist $g_{n} \in G$ and $s_{n} \in S_{n}$ such that $x=g_{n} s_{n}$. Then $s_{n}=g_{n}^{-1} x$, and we have that $d\left(s_{n}, a\right) \rightsquigarrow 0$, i.e., $d\left(g_{n}^{-1} x, a\right) \rightsquigarrow 0$. Since the orbit $G(x)$ is closed in $X$, we infer that $a \in G(x)$, or equivalently, $x \in G(a)$. Thus $p^{-1}\left(\Omega_{t}\right)=G(a)$, proving the claim.

If some $p^{-1}\left(\Omega_{t}\right)$ contains only one orbit $G(y)$ and is a tubular set with a slicing subgroup $H^{x} \subset G$, then the $p^{-1}\left(\Omega_{t}\right)$ is a subset of some $G\left(S_{x}\right)$, where $S_{x}$ is the $H^{x}$-slice described at the beginning of the proof which satisfies the condition $Q_{x} S_{x} \subset B\left(x, \varepsilon_{x}\right)$. Then the intersection $A_{x}=S_{x} \cap p^{-1}\left(\Omega_{t}\right)$ is a global $H^{x}$-slice for $p^{-1}\left(\Omega_{t}\right)$. In this case we assign to $p^{-1}\left(\Omega_{t}\right)$ the tubular segment $O_{t} R_{t}$ where $O_{t}=Q_{x}$ and $R_{t}=A_{x}$. Evidently, $\operatorname{diam} O_{t} R_{t} \leq 2 \varepsilon_{x}$.

If $p^{-1}\left(\Omega_{t}\right)$ contains more than one orbit then $\xi_{t}>0$ according to the claim above. In this case we assign to $p^{-1}\left(\Omega_{t}\right)$ a tubular segment $O_{t} R_{t} \in \mathcal{L}_{t}$ for which $\operatorname{diam} O_{t} R_{t}<2 \xi_{t}$, where $R_{t}$ is a global $H_{t}$-slice of $p^{-1}\left(\Omega_{t}\right)$, and the slicing group $H_{t}$ is a compact large subgroup of $G$.

Now we define $\mathcal{U}$ to be the totality of all these tubular segments

$$
\left\{g O_{t} R_{t} \mid g \in G, t \in T\right\} .
$$


Since the orbit space $X / G$ is paracompact the open invariant cover $p^{-1}(\omega)$ of $X$ admits a locally finite partition of unity subordinated to $p^{-1}(\omega)$; thus $\mathcal{U}$ is a $G$-normal cover. Let us check that $\mathcal{U}$ is a star-refinement of $\mathcal{V}$.

Indeed, let $g_{0} O_{t_{0}} R_{t_{0}} \in \mathcal{U}$ and $g O_{t} R_{t} \subset S t\left(g_{0} O_{t_{0}} R_{t_{0}}, \mathcal{U}\right)$, where $S t\left(g_{0} O_{t_{0}} R_{t_{0}}, \mathcal{U}\right)$ denotes the star of $g_{0} O_{t_{0}} R_{t_{0}}$ with respect to $\mathcal{U}$.

If $\xi_{t_{0}}=0$ then $R_{t_{0}}$ is a singleton $\{y\}$, and since $p^{-1}(\omega)$ is an essential cover, we infer that $\operatorname{St}\left(G\left(R_{t_{0}}\right), p^{-1}(\omega)\right)=G\left(R_{t_{0}}\right)$. Then

$$
S t\left(g_{0} O_{t_{0}} R_{t_{0}}, \mathcal{U}\right)=\bigcup\left\{g O_{t_{0}} R_{t_{0}} \mid g O_{t_{0}} R_{t_{0}} \cap g_{0} O_{t_{0}} R_{t_{0}} \neq \emptyset\right\} .
$$

Since $d$ is a $G$-invariant metric, one has diam $g O_{t_{0}} R_{t_{0}}=\operatorname{diam} O_{t_{0}} R_{t_{0}} \leq 2 \varepsilon_{x}$ which yields that $\operatorname{diam} S t\left(g_{0} O_{t_{0}} R_{t_{0}}, \mathcal{U}\right) \leq 4 \varepsilon_{x}$. Since $g_{0} y \in g_{0} S_{x} \subset B\left(g_{0} x, \varepsilon_{x}\right)$ we see that

$$
g_{0} y \in S t\left(g_{0} O_{t_{0}} R_{t_{0}}, \mathcal{U}\right) \cap B\left(g_{0} x, \varepsilon_{x}\right)
$$

which implies, in turn, that $S t\left(g_{0} O_{t_{0}} R_{t_{0}}, \mathcal{U}\right) \subset B\left(g_{0} x, 5 \varepsilon_{x}\right)$. By the $G$-invariance of the metric $d$ one has $B\left(g_{0} x, 5 \varepsilon_{x}\right)=g_{0} B\left(x, 5 \varepsilon_{x}\right)$. Now, since $B\left(x, 5 \varepsilon_{x}\right) \subset V_{x}$ with $V_{x} \in$ $\mathcal{V}$, we see that $g_{0} B\left(x, 5 \varepsilon_{x}\right) \subset g_{0} V_{x}$. But $\mathcal{V}$ is a $G$-cover, and hence, $g_{0} V_{x} \in \mathcal{V}$. Thus $S t\left(g_{0} O_{t_{0}} R_{t_{0}}, \mathcal{U}\right) \subset g_{0} V_{x}$, as required.

Now assume that $\xi_{t_{0}}>0$ and $g O_{t} R_{t} \subset S t\left(g_{0} O_{t_{0}} R_{t_{0}}, \mathcal{U}\right)$. Then $\xi_{t}>0$ and $G\left(R_{t}\right) \subset$ $\operatorname{St}\left(G\left(R_{t_{0}}\right), p^{-1}(\omega)\right)$.

Since $p^{-1}(\omega)$ is a star-refinement of $G(\mathcal{W})$, there is an element $G\left(S_{x}\right) \in G(\mathcal{W})$ such that $S t\left(G\left(R_{t_{0}}\right), p^{-1}(\omega)\right) \subset G\left(S_{x}\right)$. In particular, $G\left(R_{t}\right) \subset G\left(S_{x}\right)$. Evidently, the intersection $A=S_{x} \cap G\left(R_{t}\right)$ is a global $H^{x}$-slice of $G\left(R_{t}\right)$ and $Q_{x} A$ is a tubular segment of $G\left(R_{t}\right)$. Hence, $Q_{x} A \in \mathcal{L}_{t}$.

Next, since $Q_{x} S_{x} \subset B\left(x, \varepsilon_{x}\right)$ and $\operatorname{diam} Q_{x} A \leq \operatorname{diam} Q_{x} S_{x}$ we have that $\operatorname{diam} Q_{x} A \leq$ $2 \varepsilon_{x}$, and hence, $\xi_{t} \leq 2 \varepsilon_{x}$. Since $\xi_{t}>0$, this implies that $\operatorname{diam} O_{t} R_{t}<2 \xi_{t} \leq 4 \varepsilon_{x}$.

On the other hand, since $G\left(R_{t}\right) \subset G\left(S_{x}\right)$ we infer that $O_{t} R_{t} \cap h S_{x} \neq \emptyset$ for some $h \in G$. But $S_{x} \subset B\left(x, \varepsilon_{x}\right)$ by construction, and since $d$ is a $G$-invariant metric, this yields that $h S_{x} \subset B\left(h x, \varepsilon_{x}\right)$. Thus, $O_{t} R_{t} \cap B\left(h x, \varepsilon_{x}\right) \neq \emptyset$ and $\operatorname{diam} O_{t} R_{t}<4 \varepsilon_{x}$ which yield that $O_{t} R_{t} \subset B\left(h x, 5 \varepsilon_{x}\right)$. Again, using the $G$-invariance of the metric $d$ we get that $B\left(h x, 5 \varepsilon_{x}\right)=h B\left(x, 5 \varepsilon_{x}\right)$. But remember that $B\left(x, 5 \varepsilon_{x}\right) \subset V_{x}$ where $V_{x} \in \mathcal{V}$. Consequently, $O_{t} R_{t} \subset h B\left(x, 5 \varepsilon_{x}\right) \subset h V_{x}$ which yields that $g O_{t} R_{t} \subset g h V_{x}$. Thus, we have proved that $S t\left(g_{0} O_{t_{0}} R_{t_{0}}, \mathcal{U}\right) \subset g h V_{x}$.

Since $\mathcal{V}$ is a $G$-cover we see that $g h V_{x} \in \mathcal{V}$, and we conclude that $\mathcal{U}$ is a star-refinement of $\mathcal{V}$. This proves the first part of the lemma.

If, in addition, $X$ is a rich $G$-space then we apply once more Approximate Slice Theorem (see Theorem 2.2) in order to represent each tubular set $G\left(R_{t}\right)$ as a union of rich tubular sets, i.e.,

$$
G\left(R_{t}\right)=\bigcup_{j \in J} G\left(B_{j}\right)
$$

where each $B_{j}$ is an $H_{j}$-slice corresponding to a compact large subgroup $H_{j} \subset G$ such that $H_{j}=G_{z_{j}}$ for some point $z_{j} \in B_{j}$. Moreover, one can achieve that for each $j \in J$ there exists a neighborhood $M_{j}$ of the unity of $G$ such that $M_{j} B_{j} \subset O_{t} R_{t}$ whenever the equation (1) holds. This can be done just by repeating the argument at the beginning of this proof. 
Indeed, for each orbit $G(x) \subset G\left(R_{t}\right)$ we choose a point in the intersection $G(x) \cap O_{t} R_{t}$, say $x \in G(x) \cap O_{t} R_{t}$. Then by continuity of the action of $G$ at the point $x$, there exist a neighborhood $M_{x}$ of the unity in $G$ and a neighborhood $D_{x}$ of $x$ in $X$ such that $M_{x} D_{x} \subset O_{t} R_{t}$. Since $X$ is a rich $G$-space, by Approximate Slice Theorem (see the second part of Theorem 2.2), there exist a compact large subgroup $H^{x} \subset G$ and an $H^{x}$-slice $B_{x}$ such that $x \in B_{x} \subset D_{x}$ and $H^{x}$ is the stabilizer of some point $z \in B_{x}$. Thus $M_{x} B_{x} \subset O_{t} R_{t}$, and hence, the required representation (1) is achieved .

Now we denote by $\mathcal{Z}$ the totality of all rich tubular segments $\left\{g N_{i} B_{i} \mid g \in G, i \in I\right\}$ which arise in this way. Clearly, $\mathcal{Z}$ is an open $G$-cover of $X$ which is a refinement of $\mathcal{U}$, and hence, a star-refinement of $\mathcal{V}$. Moreover, the associated invariant open cover $\left\{G\left(B_{i}\right) \mid\right.$ $i \in I\}$ admits a subordinated locally finite partition of unity because the orbit space $X / G$ is paracompact. Thus $\mathcal{Z}$ is the desired $G$-normal cover. This completes the proof.

Notice that for the existence of a $G$-normal cover the only assumption needed is that $X$ in $G$-M. In the following proposition we additionally assume that $X$ is a proper $G$-ANR, in which case $X$ is always a rich $G$-space and thus we are able to obtain a rich $G$-normal cover.

Proposition 5.2. Let $G$ be a locally compact group and $X$ a proper $G$-ANR. Then, for any open cover $\mathcal{V}$ of $X$ there exists a rich $G$-normal cover

$$
\mathcal{U}=\left\{g O_{\mu} S_{\mu} \mid g \in G, \mu \in \mathcal{M}\right\}
$$

of $X$ such that $X$ is $\mathcal{V}$-G-dominated by the $G$-nerve $K(\mathcal{U})$ of $\mathcal{U}$.

Proof. By [2, Theorem 3.10] we can assume that $X$ is a closed invariant subset of a normed linear $G$-space $L$, and $X$ has a $G$-invariant neighbourhood $Z$ on which the action is proper. Since $X$ is a $G$-ANR, there exists a $G$-retraction $r: U \rightarrow X$, where $U$ is an invariant neighborhood of $X$ in $Z$.

Let $\mathcal{C}$ be an open cover of $U$ which refines its open cover $\left\{r^{-1}(V)\right\}_{V \in \mathcal{V}}$ and consists of open balls of $Z$. By [6, Proposition 3.10], $X$ is a rich $G$-space, and thus, by Lemma 5.1 there exists a rich $G$-normal cover

$$
\mathcal{U}=\left\{g O_{\mu} S_{\mu} \mid g \in G, \mu \in \mathcal{M}\right\}
$$

which is a star-refinement of $\mathcal{C}$.

Denote by $\Lambda=G \times \mathcal{M}$ the index set of the cover, and $U_{\lambda}=g O_{\mu} S_{\mu}$ for $\lambda=(g, \mu)$. Then we have $\mathcal{U}=\left\{U_{\lambda}\right\}_{\lambda \in \Lambda}$ and $\Lambda / G \approx \mathcal{M}$. Let $f_{\mu}: G\left(S_{\mu}\right) \rightarrow G / H_{\mu}, \mu \in \mathcal{M}=\Lambda / G$, be the corresponding slicing maps. Choose an invariant locally finite partition of unity $\left\{\varphi_{\mu}\right\}_{\mu \in \mathcal{M}}$, subordinated to the invariant cover $\widetilde{\mathcal{U}}=\left\{G\left(S_{\mu}\right)\right\}_{\mu \in \mathcal{M}}$. Let $K(\mathcal{U})$ be the $G$-nerve of $\mathcal{U}$, and $P: X \rightarrow K(\mathcal{U})$ the $G$-map defined in Proposition 4.3.

Next we define a continuous $G$-map $q^{\prime}: K(\mathcal{U}) \rightarrow U$.

For each $\mu \in \mathcal{M}$ we select a point $s_{\mu} \in S_{\mu}$ such that $G_{s_{\mu}}=H_{\mu}$ (this is possible because $\mathcal{U}$ is a rich $G$-normal cover). Define

$$
\psi_{\mu}: G / H_{\mu} \rightarrow G\left(s_{\mu}\right) ; \quad \psi_{\mu}\left(g H_{\mu}\right)=g s_{\mu} .
$$

The condition $H_{\mu} \subset G_{s_{\mu}}$ guarantees that the map $\psi_{\mu}$ is a well defined $G$-map. Then the maps $\psi_{\mu}$ and $f_{\mu} \mid: G\left(s_{\mu}\right) \rightarrow G / H_{\mu}$ are homeomorphisms inverse to each other. Define 
$q^{\prime}: K(\mathcal{U}) \rightarrow U$ by

$$
q^{\prime}(x)=\sum_{i=0}^{n} t_{i} \psi_{\mu_{i}}\left(x_{\mu_{i}}\right)
$$

where $x=\left|x_{\mu_{0}}, \ldots, x_{\mu_{n}} ; t_{0}, \ldots, t_{n}\right| \in K(\mathcal{U})$.

Clearly $q^{\prime}(K(\mathcal{U})) \subset Z$. We claim that $q^{\prime}(K(\mathcal{U})) \subset U$. Indeed, by definition there exist $\left\{U_{\lambda_{0}}, \ldots, U_{\lambda_{n}}\right\} \subset \mathcal{U}$ such that $\lambda_{i} \in \mu_{i}, x_{\mu_{i}} \in f_{\mu_{i}}\left(U_{\lambda_{i}}\right) \subset G / H_{\mu_{i}}, i=0, \ldots, n$ and $\bigcap_{i=0}^{n} U_{\lambda_{i}} \neq \emptyset$. Choose $y \in \bigcap_{i=0}^{n} U_{\lambda_{i}}$ and denote $y_{\mu_{i}}=\psi_{\mu_{i}}\left(x_{\mu_{i}}\right) \in U_{\lambda_{i}} \cap G\left(s_{\mu_{i}}\right)$. Then we have that $y \in \bigcup_{i=0}^{n} U_{\lambda_{i}} \subset \operatorname{St}(y, \mathcal{U})$. Since $\mathcal{U}$ is a star-refinement of $\mathcal{C}$, there exists a $C \in \mathcal{C}$ such that

$$
\left\{y_{\mu_{0}}, \ldots, y_{\mu_{n}}\right\} \subset \operatorname{St}(y, \mathcal{U}) \subset C .
$$

Since $C$ is convex, we have

$$
q^{\prime}(x)=\sum_{i=0}^{n} t_{i} y_{\mu_{i}} \in C \subset U
$$

which proves the claim.

Next, define

$$
q=r \circ q^{\prime}: K(\mathcal{U}) \rightarrow U \rightarrow X
$$

and a $\mathcal{V}$-G-homotopy $h: q \circ P \simeq_{G} \operatorname{id}_{X}$ by

$$
h(x, t)=r\left(t x+(1-t)\left(q^{\prime} \circ P(x)\right)\right), x \in X, t \in I .
$$

We have to verify that $t x+(1-t)\left(q^{\prime} \circ P(x)\right) \in U$. Let $x \in X$ and $\left\{\mu_{0}, \ldots, \mu_{n}\right\}=\{\mu \in$ $\left.\mathcal{M} \mid \varphi_{\mu}(x)>0\right\}$. Then there exist $\left\{U_{\lambda_{0}}, \ldots, U_{\lambda_{n}}\right\} \subset \mathcal{U}$ such that $\lambda_{i} \in \mu_{i}, i=0, \ldots, n$, and $x \in \bigcap_{i=0}^{n} U_{\lambda_{i}}$. For each $i=0, \ldots, n$, we have $\psi_{\mu_{i}} \circ f_{\mu_{i}}(x) \in U_{\lambda_{i}}$ and thus there exists a $C \in \mathcal{C}$ such that

$$
\left\{x, \psi_{\mu_{0}} f_{\mu_{0}}(x), \ldots, \psi_{\mu_{n}} f_{\mu_{n}}(x)\right\} \subset \bigcup_{i=0}^{n} U_{\lambda_{i}} \subset \operatorname{St}(x, \mathcal{U}) \subset C .
$$

Since $C$ is convex, we see that

$$
h(x, t)=t x+(1-t)\left(q^{\prime} \circ P(x)\right)=t x+(1-t) \sum_{i=0}^{n} \varphi_{\mu_{i}}(x)\left(\psi_{\mu_{i}} f_{\mu_{i}}(x)\right) \in C \subset U .
$$

Since there exists a $V \in \mathcal{V}$ with $C \subset r^{-1}(V)$, we clearly have $h(x, t) \in r(C) \subset V$ for every $t \in I$.

TheOREm 5.3. The $G$-nerve $K(\mathcal{U})$ of a $G$-normal cover of a proper $G$-space $X$ has the $G$-homotopy type of a $G$-CW complex.

Proof. We first prove that each space $K_{n}(\mathcal{U})$ is a proper $G$-CW complex. Indeed, one has $K_{n}(\mathcal{U})=\bigsqcup_{\sigma \in N_{n}} K_{\sigma}$, where each $K_{\sigma}$ is an open $G$-subset of a $G$-space of the form $G / H_{\mu_{0}} \times \cdots \times G / H_{\mu_{n}}$, where $H_{\mu_{0}}, \ldots, H_{\mu_{n}}$ are compact large subgroups of $G$ (see the definition of a $G$-nerve in Section 4$)$. Let $N_{0}, \ldots, N_{n}$ be closed normal subgroups of $G$ such that for every $i=0, \ldots, n, N_{i} \subset H_{i}$ and $G / N_{i}$ is a Lie group. If we denote $N:=N_{0} \cap \cdots \cap N_{n}$, then by Theorem 2.6, the $G / N$-space $G / H_{\mu_{0}} \times \cdots \times G / H_{\mu_{n}}$ is a proper smooth $G / N$-manifold, and hence, $K_{\sigma}$ is a proper smooth $G / N$-manifold. Thus, it follows from [23] that $K_{\sigma}$ is a locally compact $(G / N)$-CW complex, and hence, $K_{n}(\mathcal{U})$ 
is a locally compact $(G / N)$-CW complex as well. This implies, by Proposition 3.11 , that $K_{n}(\mathcal{U})$ is a locally compact $G$-CW complex.

By Proposition 3.7, $\left(K_{n}(\mathcal{U}) \times \Delta^{n}, K_{n}(\mathcal{U}) \times \partial \Delta^{n}\right)$ is a proper $G$-CW complex pair $\left(A_{n}, B_{n}\right)$. Now $K^{0}$ is a proper $G$-CW complex, and by Proposition 4.2 , for every $n=$ $1,2, \ldots, K^{n}(\mathcal{U})$ can be regarded as an adjunction space $K^{n}(\mathcal{U})=A_{n} \cup_{\alpha_{n}} K^{n-1}(\mathcal{U})$, where $A_{n}=K_{n}(\mathcal{U}) \times \Delta^{n}$ and $\alpha_{n}: B_{n}=K_{n}(\mathcal{U}) \times \partial \Delta^{n} \rightarrow K^{n-1}(\mathcal{U})$ is the $G$-map defined before Proposition 4.1. The $G$-nerve $K(\mathcal{U})=\bigcup_{n=0}^{\infty} K^{n}(\mathcal{U})$ has the weak topology with respect to the family $\left\{K^{n}(\mathcal{U})\right\}_{n=1}^{\infty}$, and thus, the claim follows from Theorem 3.9.

Recall that a map $f: X \rightarrow Y$ between topological spaces is called an $n$-equivalence for $n \geq 1$ if:

1. $f$ induces a one-to-one correspondence between the path components of $X$ and $Y$,

2. for every $x \in X$ the induced map between homotopy groups

$$
f_{*}: \pi_{q}(X, x) \rightarrow \pi_{q}(Y, f(x))
$$

is an isomorphism for $0<q<n$

3. $f_{*}$ is an epimorphism for $q=n$.

If $f$ is an $n$-equivalence for all $n \geq 1, f$ is called a weak homotopy equivalence.

Also recall from [21, p. 35] that a $G$-map $f: X \rightarrow Y$ between $G$-spaces is called an equivariant $n$-equivalence if the induced maps $f^{H}: X^{H} \rightarrow Y^{H}$ between $H$-fixed point sets are ordinary $n$-equivalences for every closed subgroup $H \leq G$. If $f$ is an equivariant $n$-equivalence for all $n \geq 1, f$ is called a weak $G$-homotopy equivalence. In what follows we will need the following Equivariant Whitehead Theorem proved in [21, Corollary I.3.7, p. 37] and [28, Theorem 5.3], see also [27, Theorem 2.4]:

Theorem 5.4. A $G$-map between $G$-CW complexes is a $G$-homotopy equivalence if and only if it is a weak G-homotopy equivalence.

The proof of the next theorem is similar to the proof of [16, Theorem 4.18].

THEOREM 5.5. Any proper $G$-space which is $G$-dominated by a $G$-CW complex has the $G$-homotopy type of a proper $G$-CW complex.

Proof of Main Theorem. We have proved in Proposition 5.2 that $X$ is $G$-dominated by the $G$-nerve $K(\mathcal{U})$ of a $G$-normal cover $\mathcal{U}$ of $X$. By Theorem $5.3, K(\mathcal{U})$ has the $G$-homotopy type of a $G$-CW complex. Thus, $X$ is dominated by a $G$-CW complex, and the theorem follows from Theorem 5.5.

Proof of Corollary 1.2. By Main Theorem, there exist $G$-homotopy equivalences $\varphi: Z \rightarrow$ $X$ and $\psi: Y \rightarrow W$, where $Z$ and $W$ are some $G$-CW complexes. This yields that for each closed subgroup $H \subset G$ the restrictions $\varphi^{H}: Z^{H} \rightarrow X^{H}$ and $\psi: Y^{H} \rightarrow W^{H}$ are ordinary homotopy equivalences. Define a $G$-map $\xi: Z \rightarrow W$ to be the composition $\psi f \varphi$. Since $\xi^{H}=\psi^{H} f^{H} \varphi^{H}$, we see that $\xi^{H}$ is a homotopy equivalence for every closed subgroup $H \subset G$, and hence, $f$ is weak $G$-homotopy equivalence. Now, in view of Equivariant Whitehead Theorem 5.4, $\xi$ should be a $G$-homotopy equivalence. Since $\varphi$ and $\psi$ are also $G$-homotopy equivalences, we conclude that $f$ is so, completing the proof. 
Proof of Corollary 1.3. Due to a result of H. Abels [1, Theorem 4.4] it suffices to prove that $X$ is a $K$-AR for every compact subgroup $K \subset G$. Since $X$, considered as a $K$-space (with the restricted $K$-action), is a $K$-ANR the result follows from its particular case corresponding to the compact group actions which was established in [7].

6. G-CW approximations. In this section we prove an equivariant version of the CW approximation theorem, see for example [31, p. 75]. For the equivariant case of compact Lie groups see [35, Theorem 3.7]. Then Theorem 5.5 can be given another proof using this $G$-CW approximation theorem and the equivariant Whitehead theorem, as in the proof of [35, Lemma 4.7].

The proof of the following theorem follows the lines given in [31], we only present how the equivariant case differs from the classical one.

TheOrem 6.1. Let $G$ be a locally compact Hausdorff group and $X$ any $G$-space. Then there is a $G$-CW complex $\Gamma X$ and a weak $G$-homotopy equivalence $\gamma: \Gamma X \rightarrow X$ (called a $G$-CW approximation). For a $G$-map $f: X \rightarrow Y$ and a $G$-CW approximation $\gamma^{\prime}: \Gamma Y \rightarrow$ $Y$ there is a G-map $\Gamma f: \Gamma X \rightarrow \Gamma Y$, unique up to G-homotopy, such that the diagram

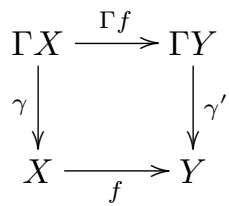

is G-homotopy commutative.

Proof. In the non-equivariant case it is obvious that we may assume that the space $X$ is path connected. Since here we have to look at all fixed point sets, the corresponding assumption cannot be made.

We denote $\mathcal{H}=\left\{H \leq G \mid X^{H} \neq \emptyset\right\}$. For each $H \in \mathcal{H}$ we choose one base point $x_{H, \alpha}$ from each path component $\alpha$ of $X^{H}$. Then we define $J_{H, \alpha}^{q}$ to be a set of representatives for generators for the homotopy group $\pi_{q}\left(X^{H}, x_{H, \alpha}\right)$, where $q \geq 1, H \in \mathcal{H}$ and $\alpha \in \pi_{0}\left(X^{H}\right)$. Thus an element $j \in J_{H, \alpha}^{q}$ is a function $j:\left(S^{q}, *\right) \rightarrow\left(X^{H}, x_{H, \alpha}\right)$. If $\pi_{q}\left(X^{H}, x_{H, \alpha}\right)=0$ for some $H, \alpha$, define $J_{H, \alpha}^{q}$ to consist of the constant map $x_{H, \alpha}$.

We define

$$
X_{0}=\coprod_{H \in \mathcal{H}} \coprod_{\alpha \in \pi_{0}\left(X^{H}\right)} \bigvee_{(q, j)} G / H \times S_{j}^{q}
$$

where the wedge is taken over pairs $(q, j)$, where $q \geq 1$ and $j \in J_{H, \alpha}^{q}$. We choose base points $y_{H, \alpha}=(e H, *) \in G / H \times S_{j}^{q}$. A $G$-map $\gamma_{0}: X_{0} \rightarrow X$ can be defined by $\left.\gamma_{0}\right|_{e H \times S_{j}^{q}}=j$.

It is clear that $\gamma_{0}$ induces epimorphisms $\left(\gamma_{0}\right)_{*}^{H}: \pi_{0}\left(X_{0}^{H}\right) \rightarrow \pi_{0}\left(X^{H}\right)$ for all $H \in \mathcal{H}$ and epimorphisms $\left(\gamma_{0}\right)_{*}^{H}: \pi_{q}\left(X_{0}^{H}, y_{H, \alpha}\right) \rightarrow \pi_{q}\left(X^{H}, x_{H, \alpha}\right)$ for all $q \geq 1, H \in \mathcal{H}$. It is also clear that $X_{0}$ has the structure of a $G$-CW complex with the base points belonging to the 0-skeleton.

If $H \in \mathcal{H}$ and $\alpha, \beta \in \pi_{0}\left(X_{0}^{H}\right)$ are such that $\alpha \neq \beta$ but $\left(\gamma_{0}\right)_{*}^{H}(\alpha)=\left(\gamma_{0}\right)_{*}^{H}(\beta)$, we attach a $G$-1-cell to $X_{0}$ by the attaching map $\varphi_{H, \alpha, \beta}: G / H \times S^{0} \rightarrow X_{0},(g H,-1) \mapsto g y_{H, \alpha}$, $(g H, 1) \mapsto g y_{H, \beta}$. Define $X_{1}$ to be the $G$-space obtained from $X_{0}$ by attaching these 
$G$-1-cells. Since the attaching maps are skeletal $\left(G / H \times D^{1}\right.$ is given the obvious $G$-CW structure) we have that $X_{1}$ is a $G$-CW complex. Since $\left(\gamma_{0}\right)_{*}^{H}(\alpha)=\left(\gamma_{0}\right)_{*}^{H}(\beta)$ above, the map $\gamma_{0}$ can be extended to give $\gamma_{1}: X_{1} \rightarrow X$, with the property that $\left(\gamma_{1}\right)^{H}$ induces bijections between the path components and epimorphisms between the homotopy groups.

As in [31] we proceed to construct a sequence $X_{0}, X_{1}, X_{2}, \ldots$ of $G$-CW complexes and $G$-maps $\gamma_{n}: X_{n} \rightarrow X$ with the corresponding properties. Then the $G$-CW complex $\Gamma X$ and the weak $G$-homotopy equivalence $\gamma: \Gamma X \rightarrow X$ are obtained as a colimit as in [31], compare also [35].

Acknowledgements. The first author was supported in part by grant IN102608 from PAPIIT (UNAM).

\section{References}

[1] H. Abels, A universal proper G-space, Math. Z. 159 (1978), 143-158.

[2] N. Antonyan, S. Antonyan and L. Rodríguez-Medina, Linearization of proper group actions, Topology Appl., to appear.

[3] S. A. Antonian, Equivariant embeddings into $G$-AR's, Glasnik Matematički 22 (42) (1987), 503-533.

[4] S. A. Antonyan, Existence of a slice for arbitrary compact transformation groups, Matematicheskie Zametki 56 (1994), no. 5, 3-9 (in Russian); English transl.: Math. Notes 56 (1994), 1101-1104.

[5] S. A. Antonyan, Extensorial properties of orbit spaces of proper group actions, Topology Appl. 98 (1999), 25-46.

[6] S. A. Antonyan, Orbit spaces and unions of equivariant absolute neighbourhood extensors, Topology Appl. 146-147 (2005), 289-315.

[7] S. A. Antonyan, G-ANR's with homotopy trivial fixed point sets, Fund. Math. 197 (2007), $1-16$.

[8] S. A. Antonyan and S. de Neymet, Invariant pseudometrics on Palais proper G-spaces, Acta Math. Hung. 98 (2003), 41-51.

[9] S. A. Antonyan and E. Elfving, The equivariant homotopy type of G-ANR's for compact group actions, Manuscripta Math. 124 (2007), 275-297.

[10] N. P. Bhatia and G. P. Szegö, Stability theory of Dynamical Systems, Springer Verlag, 1970.

[11] G. E. Bredon, Introduction to Compact Transformation Groups, Academic Press, 1972.

[12] T. tom Dieck, Partitions of unity in homotopy theory, Compositio Math. 23 (1971), 159167.

[13] T. tom Dieck, Transformation Groups, Walter de Gruyter, 1987.

[14] A. Dold, Lectures on Algebraic Topology, Springer Verlag, 1980.

[15] J. Dugundji, Topology, Allyn and Bacon, 1966.

[16] E. Elfving, The G-homotopy type of proper locally linear G-manifolds, Ann. Acad. Sci. Fenn. Math. Dissertationes 108 (1996).

[17] E. Elfving, The G-homotopy type of proper locally linear G-manifolds. II, Manuscripta Math. 105 (2001), 235-251.

[18] R. Engelking, General Topology, PWN-Pol. Sci. Publ., Warsaw, 1977. 
[19] S. Helgason, Differential Geometry, Lie Groups and Symmetric Spaces, Graduate Studies in Mathematics 34, Amer. Math. Soc., Providence, R.I., 2001.

[20] S. Illman, Equivariant singular homology and cohomology for actions of compact Lie groups, in: Proceedings of the Second Conference on Compact Transformation Groups (Amherst, 1971), Lecture Notes in Mathematics 298, Springer, 1972, 403-415.

[21] S. Illman, Equivariant algebraic topology, Ph.D. Thesis, Princeton University, Princeton, N.J., 1972.

[22] S. Illman, Restricting the transformation group in equivariant CW complexes, Osaka J. Math. 27 (1990), 191-206.

[23] S. Illman, Existence and uniqueness of equivariant triangulations of smooth proper $G$ manifolds with some applications to equivariant Whitehead torsion, J. Reine Angew. Math. 524 (2000), 129-183.

[24] I. M. James and G. B. Segal, On equivariant homotopy type, Topology 17 (1978), 267-272.

[25] I. M. James and G. B. Segal, On equivariant homotopy theory, in: Proceedings of the Topology Symposium (Siegen, 1979), Lecture Notes in Mathematics 788, Springer, 1980, 316-330.

[26] J. L. Koszul, Lectures on groups of transformations, Tata Institute of Fundamental Research, Bombay, 1965.

[27] W. Lück, Transformation Groups and Algebraic K-theory, Lecture Notes in Mathematics 1408, Springer, 1989.

[28] T. Matumoto, On G-CW complexes and a theorem of J.H.C. Whitehead, J. Fac. Sci. Univ. Tokyo Sect. IA Math. 18 (1971), 363-374.

[29] T. Matumoto, Equivariant K-theory and Fredholm operators, J. Fac. Sci. Univ. Tokyo Sect. IA Math. 18 (1971), 109-125.

[30] J. P. May, Simplicial Objects in Algebraic Topology, D. van Nostrand Company, 1967.

[31] J. P. May, A Concise Course in Algebraic Topology, Chicago Lectures in Mathematics, The University of Chicago Press, 1999.

[32] R. Palais, On the existence of slices for actions of non-compact Lie groups, Ann. of Math. 73 (1961), 295-323.

[33] G. Segal, Classifying spaces and spectral sequences, Publ. Math. I.H.E.S. 34 (1968), 105112.

[34] E. H. Spanier, Algebraic Topology, McGraw-Hill, 1966.

[35] S. Waner, Equivariant homotopy theory and Milnor's theorem, Trans. Amer. Math. Soc. 258 (1980), 351-368. 